\title{
Gravitational perturbations of the Schwarzschild spacetime: A practical covariant and gauge-invariant formalism
}

\author{
Karl Martel and Eric Poisson \\ Department of Physics, University of Guelph, Guelph, Ontario, Canada N1G 2W1
}

(Dated: January 23, 2004)

\begin{abstract}
We present a formalism to study the metric perturbations of the Schwarzschild spacetime. The formalism is gauge invariant, and it is also covariant under two-dimensional coordinate transformations that leave the angular coordinates unchanged. The formalism is applied to the typical problem of calculating the gravitational waves produced by material sources moving in the Schwarzschild spacetime. We examine the radiation escaping to future null infinity as well as the radiation crossing the event horizon. The waveforms, the energy radiated, and the angular-momentum radiated can all be expressed in terms of two gauge-invariant scalar functions that satisfy one-dimensional wave equations. The first is the Zerilli-Moncrief function, which satisfies the Zerilli equation, and which represents the even-parity sector of the perturbation. The second is the Cunningham-Price-Moncrief function, which satisfies the Regge-Wheeler equation, and which represents the odd-parity sector of the perturbation. The covariant forms of these wave equations are presented here, complete with covariant source terms that are derived from the stress-energy tensor of the matter responsible for the perturbation. Our presentation of the formalism is concluded with a separate examination of the monopole and dipole components of the metric perturbation.
\end{abstract}

\section{INTRODUCTION}

Metric perturbations of the Schwarzschild spacetime have been studied for a long time, starting with the pioneering work of Regge and Wheeler [1], Vishveshwara 2], and Zerilli [3]. The theory was summarized in an influential monograph by Chandrasekhar [4], and it has been applied to many different physical situations (see, for example, Chapter 4 of the book by Frolov and Novikov [5]). In particular, a useful application has been the computation of gravitational waves produced by a point particle moving in the field of a Schwarzschild black hole (see Ref. 6], the review article of Ref. 7], and references therein). Another has been the simulation of a collision of two black holes in a "close-limit" approximation (see the review article of Ref. [8] and references therein).

Traditionally the perturbation formalism is developed in the standard Schwarzschild coordinates $(t, r, \theta, \phi)$, and in a standard choice of gauge known as the "ReggeWheeler gauge." The tradition also makes use of Fouriertransform techniques and presents the perturbation equations in the frequency domain instead of the time domain. Moncrief $[\underline{9}]$ was the first to present the formalism in a gauge-invariant package, recognizing the practical advantages that gauge invariance provides: While the Regge-Wheeler gauge is useful for many purposes, it is not useful for others, and the power to switch from one gauge to another within a gauge-invariant framework is often required. Another refinement of the formalism was produced by Gerlach and Sengupta [10], who presented the gauge-invariant perturbation equations in an arbitrary coordinate system, thus liberating the formalism from the usual Schwarzschild coordinates and their poor behavior at the event horizon.

This program to translate the traditional perturbation formalism into a covariant, gauge-invariant language was recently revived by Sarbach and Tiglio [1], and with this paper we hope to deliver its final chapter. We aim to present the formalism of metric perturbations of the Schwarzschild spacetime in its most mature and practical incarnation yet. Our formalism is covariant and gauge invariant, and it goes beyond the work of Gerlach and Sengupta, and of Sarbach and Tiglio, by the development of covariant master equations for the evenparity and odd-parity sectors of the theory, complete with covariant source terms that are derived from the stressenergy tensor of the matter which is responsible for the perturbation.

We have in mind a typical application of the perturbation formalism, the calculation of gravitational waves produced by material sources moving in the Schwarzschild spacetime. We are interested in the radiation that escapes to future null infinity and manifests itself as waveforms $h_{+}$and $h_{\times}$that are directly observable to gravitational-wave detectors, and we are interested in the radiation that crosses the black-hole horizon. This application illustrates well the need for a covariant and gauge-invariant formalism, as the two types of radiation admit different descriptions. The radiation at future null infinity is best described by casting the perturbation in an outgoing radiation gauge and expressing it in a retarded coordinate system $(u, r, \theta, \phi)$ related to the usual Schwarzschild coordinates by the transformation $u=t-r-2 M \ln (r / 2 M-1)$. The radiation at the horizon, on the other hand, is best described by adopting an incoming radiation gauge and expressing the perturbation in an advanced coordinate system $(v, r, \theta, \phi)$ related to the usual Schwarzschild coordinates by the transformation $v=t+r+2 M \ln (r / 2 M-1)$. Our formalism permits the use of any coordinate system $x^{a}=\left(x^{0}, x^{1}\right)$ that can be obtained from the usual Schwarzschild coordinates $(t, r)$; we do not, however, consider transformations of the angular coordinates $\theta^{A}=(\theta, \phi)$.

In the formalism developed in this paper, the radiation 
at future null infinity and at the horizon are described in terms of scalar and gauge-invariant master functions, $\Psi_{\text {even }}^{l m}\left(x^{a}\right)$ and $\Psi_{\text {odd }}^{l m}\left(x^{a}\right)$, which can be computed from the metric perturbations. These functions are labeled by spherical-harmonic indices $l$ and $m$, and by their behavior under a parity transformation. The function $\Psi_{\text {even }}^{l m}\left(x^{a}\right)$ is constructed from the even-parity perturbations, and it is equal to the gauge-invariant function that was first introduced by Moncrief [9]; it is a close cousin to Zerilli's original master function 3], and it satisfies a covariant version of Zerilli's differential equation. The complete source term for this equation is presented for the first time in this paper. The function $\Psi_{\text {odd }}^{l m}\left(x^{a}\right)$, on the other hand, is constructed from the odd-parity perturbations, and it is equal to the gauge-invariant function that was first introduced by Cunningham, Price, and Moncrief [12] and recently revived by Jhingan and Tanaka [13]; it is essentially the time integral of the original Regge-Wheeler master function [1], and it satisfies a covariant version of the Regge-Wheeler equation. The complete source term for this equation is presented for the first time in this paper.

The paper is organized as follows. In Sec. II we give a covariant description of the Schwarzschild spacetime and specify our notations and conventions. In Sec. III we introduce the scalar, vector, and tensor spherical harmonics that are used in the decomposition of the metric perturbation. In Sec. IV we examine the even-parity sector of the perturbation, introduce the Zerilli-Moncrief master function, and derive the one-dimensional wave equation that it satisfies. In Sec. V we examine the odd-parity sector of the perturbation, introduce the CunninghamPrice-Moncrief master function, and derive the onedimensional wave equation that it satisfies. In Sec. VI we describe the behavior of the perturbations near future null infinity, construct the radiative part of the perturbation field, extract the waveforms $h_{+}$and $h_{\times}$, and compute the rates at which the radiation carries away energy and angular momentum. In Sec.VII we describe the behavior of the perturbations near the black hole's event horizon, and calculate the rates at which they transfer energy and angular momentum to the black hole. In Sec. VIII we conclude our presentation and examine the nonradiating pieces of the metric perturbation, those associated with the low multipoles $l=0$ and $l=1$.

Various technical details are relegated to the Appendices. In Appendix $\mathrm{A}$ we expand our discussion of vectorial and tensorial spherical harmonics. In Appendix B] we present the perturbed Ricci tensor for a general spherically-symmetric background spacetime. In Appendices [C] [D] and $\mathrm{E}$ we list the perturbation equations in the commonly used coordinate systems $x^{a}=(t, r)$, $x^{a}=(u, r)$, and $x^{a}=(v, r)$, respectively.

Because the topic of metric perturbations of the Schwarzschild spacetime is so venerable, we will allow ourselves in this paper to simply state our results and omit most lengthy derivations that lead to those results. We hope, however, that the path to the results will always be clearly delineated. We refer the reader to the literature reviewed in this Introduction for additional details; another repository of relevant derivations is Martel's PhD dissertation [14]. Throughout this paper we adopt the sign conventions of Misner, Thorne, and Wheeler [15], and we set $c=G=1$.

\section{SCHWARZSCHILD SPACETIME}

The Schwarzschild metric is expressed as

$$
d s^{2}=g_{a b} d x^{a} d x^{b}+r^{2} \Omega_{A B} d \theta^{A} d \theta^{B},
$$

in a form that is covariant under two-dimensional coordinate transformations $x^{a} \rightarrow x^{\prime a}$. The coordinates $x^{a}$ span the submanifold $\mathcal{M}^{2}$ of the Schwarzschild spacetime - the " $(t, r)$ plane" - and lower-case Latin indices $a$, $b$, c, etc. run over the values 0 and 1 . The coordinates $\theta^{A}=(\theta, \phi)$ span the two-spheres $x^{a}=$ constant, and upper-case Latin indices $A, B, C$, etc. run over the values 2 and 3. The full spacetime manifold is $\mathcal{M}=\mathcal{M}^{2} \times S^{2}$. The two-dimensional tensor $g_{a b}$ and the scalar $r$ are functions of the coordinates $x^{a}$, and $\Omega_{A B}=\operatorname{diag}\left(1, \sin ^{2} \theta\right)$ is the metric on the unit two-sphere.

We shall use three different coordinate systems $x^{a}$ in the applications of the perturbation formalism to be presented below. The first is $(t, r)$, the usual Schwarzschild coordinates. The second is $(u, r)$, where the retardedtime coordinate $u$ is defined by $u=t-r-2 M \ln (r / 2 M-$ $1)$. The third is $(v, r)$, where the advanced-time coordinate $v$ is defined by $v=t+r+2 M \ln (r / 2 M-1)$. In these coordinates the Schwarzschild metric takes the form

$$
\begin{aligned}
g_{a b} d x^{a} d x^{b} & =f d t^{2}+f^{-1} d r^{2} \\
& =f d u^{2}-2 d u d r \\
& =f d v^{2}+2 d v d r
\end{aligned}
$$

where $f:=1-2 M / r$ and $M$ is the mass of the black hole. These systems share the property that the scalar $r$ is adopted as one of the coordinates. Our formalism is not, however, limited to these coordinate choices; one retains the freedom of using any coordinate system whatever, for example, harmonic coordinates, isotropic coordinates, or double-null coordinates.

We introduce the dual vector

$$
r_{a}:=\frac{\partial r}{\partial x^{a}},
$$

which is normal to the surfaces of constant $r\left(x^{a}\right)$; in the coordinates of Eqs. (2.2)-(2.4), $r_{a}=(0,1)$. We use $g^{a b}$, the inverse to $g_{a b}$, to raise its index: $r^{a}=g^{a b} r_{b}$. This allows us to give a covariant definition to the function $f$ that appears in Eqs. (2.2)- (2.4):

$$
r^{a} r_{a}=: f=1-\frac{2 M}{r} \text {. }
$$

We also introduce $\varepsilon_{a b}$, the (antisymmetric) Levi-Civita tensor on $\mathcal{M}^{2}$; in the coordinates of Eqs. (2.2)-(2.4), 
$\varepsilon_{t r}=\varepsilon_{u r}=\varepsilon_{v r}=1$. The timelike Killing vector of the Schwarzschild spacetime is tangent to $\mathcal{M}^{2}$ and is given by

$$
t^{a}=-\varepsilon^{a b} r_{b}
$$

in the coordinates of Eqs. (2.2)-(2.4), $t^{a}=(1,0)$. We have $t^{a} t_{a}=-f$ and $t^{a} r_{a}=0$, and the vectors $r^{a}, t^{a}$ form a basis on $\mathcal{M}^{2}$. In terms of this basis we have $g^{a b}=f^{-1}\left(-t^{a} t^{b}+r^{a} r^{b}\right)$ and $\varepsilon^{a b}=-f^{-1}\left(t^{a} r^{b}-r^{a} t^{b}\right)$.

The covariant derivative operator compatible with $g_{a b}$ is denoted $\nabla_{a}$; we thus have $\nabla_{a} g_{b c} \equiv 0$. It is easy to show that for the Schwarzschild solution,

$$
\nabla_{a} \nabla_{b} r=\frac{M}{r^{2}} g_{a b}
$$

so that $\square r=2 M / r^{2}$, where $\square:=g^{a b} \nabla_{a} \nabla_{b}$ is the Laplacian operator on $\mathcal{M}^{2}$. We also have $\nabla_{a} t_{b}=\left(M / r^{2}\right) \varepsilon_{a b}$, which confirms that $t^{a}$ is a Killing vector. The Riemann tensor on $\mathcal{M}^{2}$ is $R_{a b c d}=\left(2 M / r^{3}\right)\left(g_{a c} g_{b d}-g_{a d} g_{b c}\right)$.

We let $\Omega^{A B}$ be the inverse to $\Omega_{A B}$, the metric on the unit two-sphere. The covariant derivative operator compatible with $\Omega_{A B}$ is denoted $D_{A}$; we thus have $D_{A} \Omega_{B C} \equiv$ 0 . The Levi-Civita tensor on the unit two-sphere is denoted $\varepsilon_{A B}$, and $\varepsilon_{\theta \phi}=\sin \theta$. The Riemann tensor on the unit-sphere is $R_{A B C D}=\Omega_{A C} \Omega_{B D}-\Omega_{A D} \Omega_{B D}$.

Covariant differentiation in the Schwarzschild spacetime can be defined in terms of covariant differentiation in the submanifolds $\mathcal{M}^{2}$ and $S^{2}$. If $\Gamma_{b c}^{a}$ is the connection associated with $\nabla_{a}$, and if $\Gamma_{B C}^{A}$ is the connection associated with $D_{A}$, then it is easy to show that the nonvanishing components of the spacetime connection are given by ${ }^{4} \Gamma_{b c}^{a}=\Gamma_{b c}^{a},{ }^{4} \Gamma^{a}{ }_{B C}=-r r^{a} \Omega_{B C},{ }^{4} \Gamma_{B c}^{A}=r^{-1} r_{c} \delta_{B}^{A}$, and ${ }^{4} \Gamma_{B C}^{A}=\Gamma_{B C}^{A}$. Using these rules we find that the conservation identities for a stress-energy tensor $T^{\alpha \beta}$ in the Schwarzschild spacetime take the form

$$
\nabla_{b} T^{a b}+D_{B} T^{a B}+\frac{2}{r} r_{b} T^{a b}-r r^{a} \Omega_{A B} T^{A B}=0
$$

and

$$
\nabla_{a} T^{a A}+D_{B} T^{A B}+\frac{4}{r} r_{a} T^{a A}=0
$$

when expressed in terms of the submanifold connections.

\section{SPHERICAL HARMONICS}

In this section we introduce the scalar, vector, and tensor spherical harmonics that are used in the decomposition of the metric perturbation. The tensorial nature of the spherical harmonics refers to the unit two-sphere, and in this section we use the metric $\Omega_{A B}$ and its inverse $\Omega^{A B}$ to lower and raise all upper-case Latin indices. All tensorial operations (including covariant differentiation) shall refer to this metric.

The scalar harmonics are the usual spherical-harmonic functions $Y^{l m}\left(\theta^{A}\right)$. They satisfy the eigenvalue equation $\left[\Omega^{A B} D_{A} D_{B}+l(l+1)\right] Y^{l m}=0$.
Vectorial spherical harmonics come in two types. The even-parity harmonics are defined by

$$
Y_{A}^{l m}:=D_{A} Y^{l m}
$$

while the odd-parity harmonics are

$$
X_{A}^{l m}:=-\varepsilon_{A}^{B} D_{B} Y^{l m} .
$$

Their components are listed explicitly in Appendix A The vectorial harmonics satisfy the orthogonality relations

$$
\int \bar{Y}_{l m}^{A} Y_{A}^{l^{\prime} m^{\prime}} d \Omega=l(l+1) \delta_{l l^{\prime}} \delta_{m m^{\prime}}
$$

and

$$
\int \bar{X}_{l m}^{A} X_{A}^{l^{\prime} m^{\prime}} d \Omega=l(l+1) \delta_{l l^{\prime}} \delta_{m m^{\prime}}
$$

in which an overbar indicates complex conjugation and $d \Omega:=\sin \theta d \theta d \phi$ is an element of solid angle. We also have

$$
\int \bar{Y}_{l m}^{A} X_{A}^{l^{\prime} m^{\prime}} d \Omega=0
$$

which states that the even-parity and odd-parity harmonics are always orthogonal. The definitions (3.1) and (3.2) for the vectorial spherical harmonics are identical to those provided by Regge and Wheeler 1].

Tensorial spherical harmonics come in the same two types. The even-parity harmonics are $\Omega_{A B} Y^{l m}$ and

$$
Y_{A B}^{l m}:=\left[D_{A} D_{B}+\frac{1}{2} l(l+1) \Omega_{A B}\right] Y^{l m},
$$

while the odd-parity harmonics are

$$
X_{A B}^{l m}:=-\frac{1}{2}\left(\varepsilon_{A}^{C} D_{B}+\varepsilon_{B}^{C} D_{A}\right) D_{C} Y^{l m} .
$$

Their components are listed explicitly in Appendix A The tensorial harmonics satisfy the orthogonality relations

$$
\int \bar{Y}_{l m}^{A B} Y_{A B}^{l^{\prime} m^{\prime}} d \Omega=\frac{1}{2}(l-1) l(l+1)(l+2) \delta_{l l^{\prime}} \delta_{m m^{\prime}}
$$

and

$$
\int \bar{X}_{l m}^{A B} X_{A B}^{l^{\prime} m^{\prime}} d \Omega=\frac{1}{2}(l-1) l(l+1)(l+2) \delta_{l l^{\prime}} \delta_{m m^{\prime}}
$$

We also have

$$
\int \bar{Y}_{l m}^{A B} X_{A B}^{l^{\prime} m^{\prime}} d \Omega=0
$$

and

$$
\Omega^{A B} Y_{A B}^{l m}=0=\Omega^{A B} X_{A B}^{l m}
$$

The definition (3.6) for the even-parity harmonics does not agree with that of Regge and Wheeler [1], who work 
instead with the set $\Omega_{A B} Y^{l m}$ and $D_{A} D_{B} Y^{l m}$. We find it more convenient to form the tracefree combinations $Y_{A B}^{l m}$, which have the property of being (pointwise) orthogonal to $\Omega_{A B} Y^{l m}$. The definition (3.7) for the odd-parity harmonics also differs from Regge and Wheeler's, but only by an overall minus sign, which we find convenient to introduce.

The tensorial harmonics $Y_{A B}^{l m}$ and $X_{A B}^{l m}$ can be related to the spherical-harmonic functions of spin-weight $s=$ \pm 2 [16], and to the pure-spin harmonics used by Thorne 17. These relations are explored in Appendix A

\section{EVEN-PARITY SECTOR}

\section{A. Perturbation fields and gauge transformations}

The even-parity sector refers to those components of the metric perturbation that can be expanded in terms of the even-parity spherical harmonics $Y^{l m}, Y_{A}^{l m}, \Omega_{A B} Y^{l m}$, and $Y_{A B}^{l m}$. Introducing the notation ${ }^{4} g_{a b}=g_{a b}+p_{a b}$, ${ }^{4} g_{a B}=p_{a B}$, and ${ }^{4} g_{A B}=r^{2} \Omega_{A B}+p_{A B}$ for the perturbed metric, the even-parity sector of the metric perturbation is

$$
\begin{aligned}
p_{a b} & =\sum_{l m} h_{a b}^{l m} Y^{l m}, \\
p_{a B} & =\sum_{l m} j_{a}^{l m} Y_{B}^{l m}, \\
p_{A B} & =r^{2} \sum_{l m}\left(K^{l m} \Omega_{A B} Y^{l m}+G^{l m} Y_{A B}^{l m}\right) .
\end{aligned}
$$

In this section the sums over $l$ are taken to begin at $l=2$; the low multipoles $(l=0$ and $l=1)$ will be considered separately in Sec. VIII The fields $h_{a b}^{l m}$, $j_{a}^{l m}, K^{l m}$, and $G^{l m}$ are defined on $\mathcal{M}^{2}$ and they depend on the coordinates $x^{a}$ only. They are closely related to the quantities first introduced by Regge and Wheeler [1], who worked exclusively in terms of the usual Schwarzschild coordinates $(t, r)$. In these coordinates (discarding for brevity the spherical-harmonic labels) we have $h_{t t}=f H_{0}, h_{t r}=H_{1}, h_{r r}=H_{2} / f, j_{t}=h_{0}$, and $j_{r}=h_{1}$. The function $G$ introduced in Eq. (4.3) is identical to the corresponding Regge-Wheeler quantity, but $K$ is different: $K_{\text {here }}=K_{\mathrm{RW}}-\frac{1}{2} l(l+1) G$; the difference originates from the fact that Regge and Wheeler work with $\Omega_{A B} Y^{l m}$ and $D_{A} D_{B} Y^{l m}$ instead of $\Omega_{A B} Y^{l m}$ and $Y_{A B}^{l m}$.

Even-parity gauge transformations are generated by a dual vector field $\Xi_{\alpha}=\left(\Xi_{a}, \Xi_{A}\right)$ that is expanded as

$$
\begin{aligned}
& \Xi_{a}=\sum_{l m} \xi_{a}^{l m} Y^{l m} \\
& \Xi_{A}=\sum_{l m} \xi^{l m} Y_{A}^{l m} ;
\end{aligned}
$$

the fields $\xi_{a}^{l m}$ and $\xi^{l m}$ depend on the coordinates $x^{a}$ only.
Under such a transformation the perturbation quantities change according to (see Appendix [B]

$$
\begin{aligned}
h_{a b} & \rightarrow h_{a b}^{\prime}=h_{a b}-\nabla_{a} \xi_{b}-\nabla_{b} \xi_{a}, \\
j_{a} & \rightarrow j_{a}^{\prime}=j_{a}-\xi_{a}-\nabla_{a} \xi+\frac{2}{r} r_{a} \xi \\
K & \rightarrow K^{\prime}=K+\frac{l(l+1)}{r^{2}} \xi-\frac{2}{r} r^{a} \xi_{a}, \\
G & \rightarrow G^{\prime}=G-\frac{2}{r^{2}} \xi
\end{aligned}
$$

where we have discarded the spherical-harmonic labels for brevity (we shall continue with this practice until the end of the section). It is easy to show that the combinations

$$
\tilde{h}_{a b}:=h_{a b}-\nabla_{a} \varepsilon_{b}-\nabla_{b} \varepsilon_{a}
$$

and

$$
\tilde{K}:=K+\frac{1}{2} l(l+1) G-\frac{2}{r} r^{a} \varepsilon_{a}
$$

are gauge invariant, where

$$
\varepsilon_{a}:=j_{a}-\frac{1}{2} r^{2} \nabla_{a} G .
$$

Equations (4.7) and (4.9) reveal that one can always choose a gauge in which $j_{a}=0=G$; this is the ReggeWheeler gauge. Equations (4.10)-4.12) imply that $\tilde{h}_{a b}=$ $h_{a b}$ and $\tilde{K}=K$ in the Regge-Wheeler gauge.

\section{B. Perturbation equations}

The Ricci tensor of the Schwarzschild spacetime vanishes, and as a consequence its perturbation is gauge invariant. Its computation can therefore be carried out in any convenient gauge, and the Regge-Wheeler gauge is clearly convenient. The steps involved are as follows. We substitute Eqs. (4.1)-(4.3), having set $j_{a}=G=0$, into the Ricci tensor of Appendix B] We simplify the result and find that $\delta R_{a b}$ is expanded in terms of $Y^{l m}, \delta R_{a B}$ in terms of $Y_{B}^{l m}$, and $\delta R_{A B}$ in terms of both $\Omega_{A B} Y^{l m}$ and $Y_{A B}^{l m}$. From the Ricci tensor we compute the Einstein tensor and set the result equal to $8 \pi T^{\alpha \beta}$, which describes the material source for the gravitational perturbations. Each spherical-harmonic component of the field equations can then be extracted by involving the orthonormality relations (3.3) and (3.8) satisfied by the spherical harmonics. At the end of this calculation we take advantage of the fact that $\tilde{h}_{a b}=h_{a b}$ and $\tilde{K}=K$ in the Regge-Wheeler gauge. This allows us to make the substitutions $h_{a b} \rightarrow \tilde{h}_{a b}, K \rightarrow \tilde{K}$ and therefore to express the gauge-invariant Einstein tensor in terms of gauge-invariant quantities.

Our final results are 


$$
\begin{aligned}
Q_{a b}= & \nabla_{c} \nabla_{(a} \tilde{h}_{b)}^{c}-\frac{1}{2} g_{a b} \nabla_{c} \nabla_{d} \tilde{h}^{c d}-\frac{1}{2} \square\left(\tilde{h}_{a b}-g_{a b} \tilde{h}\right)-\frac{1}{2} \nabla_{a} \nabla_{b} \tilde{h}+\frac{2}{r} r_{c}\left(\nabla_{(a} \tilde{h}_{b)}^{c}-g_{a b} \nabla_{d} \tilde{h}^{c d}\right) \\
& -\frac{1}{r} r^{c} \nabla_{c}\left(\tilde{h}_{a b}-g_{a b} \tilde{h}\right)+\frac{l(l+1)}{2 r^{2}} \tilde{h}_{a b}-\frac{1}{r^{2}} g_{a b} r_{c} r_{d} \tilde{h}^{c d}-\frac{1}{2}\left[\frac{l(l+1)}{r^{2}}+\frac{2 M}{r^{3}}\right] g_{a b} \tilde{h} \\
& -\nabla_{a} \nabla_{b} \tilde{K}+g_{a b} \square \tilde{K}-\frac{2}{r} r_{(a} \nabla_{b)} \tilde{K}+\frac{3}{r} g_{a b} r^{c} \nabla_{c} \tilde{K}-\frac{(l-1)(l+2)}{2 r^{2}} g_{a b} \tilde{K}, \\
Q_{a}= & \nabla_{c} \tilde{h}_{a}^{c}-\nabla_{a} \tilde{h}+\frac{1}{r} r_{a} \tilde{h}-\nabla_{a} \tilde{K}, \\
Q^{b}= & \square \tilde{h}-\nabla_{a} \nabla_{b} \tilde{h}^{a b}-\frac{2}{r} r_{a} \nabla_{b} \tilde{h}^{a b}+\frac{1}{r} r^{a} \nabla_{a} \tilde{h}-\frac{l(l+1)}{2 r^{2}} \tilde{h}+\square \tilde{K}+\frac{2}{r} r^{a} \nabla_{a} \tilde{K}, \\
Q^{\sharp}= & -\tilde{h},
\end{aligned}
$$

where $\tilde{h}:=g^{a b} \tilde{h}_{a b}$ and $\square:=g^{a b} \nabla_{a} \nabla_{b}$. The source terms are

$$
\begin{aligned}
Q^{a b} & =8 \pi \int T^{a b} \bar{Y}^{l m} d \Omega, \\
Q^{a} & =\frac{16 \pi r^{2}}{l(l+1)} \int T^{a B} \bar{Y}_{B}^{l m} d \Omega, \\
Q^{b} & =8 \pi r^{2} \int T^{A B} \Omega_{A B} \bar{Y}^{l m} d \Omega, \\
Q^{\sharp} & =\frac{32 \pi r^{4}}{(l-1) l(l+1)(l+2)} \int T^{A B} \bar{Y}_{A B}^{l m} d \Omega .
\end{aligned}
$$

In Eqs. 4.17) -4.20 the stress-energy tensor is imagined to be given in fully contravariant form; $T^{a b}, T^{a B}$, and $T^{A B}$ are then its components in the spacetime coordinates $\left(x^{a}, \theta^{A}\right)$. In the event where the stress-energy tensor would be given in covariant or mixed form, its indices would have to be raised with $\left(g^{a b}, r^{-2} \Omega^{A B}\right)-$ the inverse Schwarzschild metric - before evaluating the source terms. In Eqs. (4.13)-4.16) all lower-case Latin indices are lowered and raised with $g_{a b}$ and $g^{a b}$, respectively.

The perturbation equations are not all independent. By virtue of the Bianchi identities, or the conservation equations (2.9) and (2.10), they are related by

$$
\nabla_{b} Q^{a b}+\frac{2}{r} r_{b} Q^{a b}-\frac{l(l+1)}{2 r^{2}} Q^{a}-\frac{1}{r} r^{a} Q^{b}=0
$$

and

$$
\nabla_{a} Q^{a}+\frac{2}{r} r_{a} Q^{a}-\frac{(l-1)(l+2)}{2 r^{2}} Q^{\sharp}+Q^{b}=0 .
$$

\section{Master equation}

The Zerilli-Moncrief function is defined by

$$
\Psi_{\text {even }}^{l m}:=\frac{2 r}{l(l+1)}\left[\tilde{K}^{l m}+\frac{2}{\Lambda}\left(r^{a} r^{b} \tilde{h}_{a b}^{l m}-r r^{a} \nabla_{a} \tilde{K}^{l m}\right)\right],
$$

where

$$
\Lambda:=(l-1)(l+2)+\frac{6 M}{r} .
$$

This is a covariant and gauge-invariant generalization of the definition provided by Lousto and Price [18], who worked with the usual Schwarzschild coordinates and in the Regge-Wheeler gauge. Their even-parity function agrees, up to a normalization factor, with the gaugeinvariant function first introduced by Moncrief [9]. Moncrief's function, in turn, is a variant of Zerilli's original even-parity function [3] (Zerilli worked in the frequency domain instead of the Schwarzschild time domain). Up to the same normalization factor the Moncrief and Fouriertransformed Zerilli functions are equal up to the presence of source terms; they are equal only where there is no matter. The normalization adopted in Eq. (4.23) will be seen to be convenient when we discuss gravitational radiation at future null infinity (Sec. VI) and at the horizon (Sec. VIII); our definition of the even-parity master function is well adapted to the description of radiation fields.

The perturbation equations (4.13)-(4.16) give rise to a wave equation for the function $\Psi_{\text {even }}$ (we resume our practice of omitting the spherical-harmonic labels). The manipulations are long and tedious, and we shall not present them here (the reader may consult Ref. 14] for details). We simply state the final result: As a consequence of the field equations, the even-parity master function satisfies the Zerilli equation

$$
\left(\square-V_{\text {even }}\right) \Psi_{\text {even }}=S_{\text {even }},
$$

with potential

$$
V_{\text {even }}=\frac{1}{\Lambda^{2}}\left[\mu^{2}\left(\frac{\mu+2}{r^{2}}+\frac{6 M}{r^{3}}\right)+\frac{36 M^{2}}{r^{4}}\left(\mu+\frac{2 M}{r}\right)\right]
$$

and source term 
$S_{\text {even }}=\frac{4}{\Lambda} r_{a} Q^{a}-\frac{1}{r} Q^{\sharp}+\frac{2}{(\mu+2) \Lambda}\left\{-2 r^{2} r^{a} \nabla_{a} Q+\frac{24 M}{\Lambda} r_{a} r_{b} Q^{a b}+2 r f Q^{b}+\frac{r}{\Lambda}\left[\mu(\mu-2)+12(\mu-3) \frac{M}{r}+84 \frac{M^{2}}{r^{2}}\right] Q\right\}$,

where $\mu:=(l-1)(l+2)$ and $Q:=g_{a b} Q^{a b}$. The validity of Eqs. (4.25) 4.27) can be verified by brute-force evaluation of both sides of Eqs. 4.25). The general source term for the Zerilli equation has never been presented in the literature; we display it here for the first time, in a form covariant under an arbitrary transformation of the coordinates $x^{a}$ used in the two-dimensional submanifold $\mathcal{M}^{2}$ of the Schwarzschild spacetime.

\section{ODD-PARITY SECTOR}

\section{A. Perturbation fields and gauge transformations}

The odd-parity sector refers to those components of the metric perturbation that can be expanded in terms of the odd-parity spherical harmonics $X_{A}^{l m}$ and $X_{A B}^{l m}$. Recalling the notation ${ }^{4} g_{a b}=g_{a b}+p_{a b},{ }^{4} g_{a B}=p_{a B}$, and ${ }^{4} g_{A B}=$ $r^{2} \Omega_{A B}+p_{A B}$ for the perturbed metric, the odd-parity sector of the metric perturbation is

$$
\begin{aligned}
p_{a b} & =0, \\
p_{a B} & =\sum_{l m} h_{a}^{l m} X_{B}^{l m}, \\
p_{A B} & =\sum_{l m} h_{2}^{l m} X_{A B}^{l m} .
\end{aligned}
$$

In this section the sums over $l$ are taken to begin at $l=2$; there is no odd-parity perturbation with $l=0$, and the case $l=1$ will be considered separately in Sec. VIII The fields $h_{a}^{l m}$ and $h_{2}^{l m}$ are defined on $\mathcal{M}^{2}$ and they depend on the coordinates $x^{a}$ only. They are closely related to the quantities first introduced by Regge and Wheeler [1], who worked exclusively in terms of the usual Schwarzschild coordinates $(t, r)$. In these coordinates (discarding spherical-harmonic labels) we have $h_{t}=h_{0}$ and $h_{r}=h_{1}$. Except for a sign - refer back to the discussion following Eq. (3.11) - the function $h_{2}$ is identical to the corresponding Regge-Wheeler quantity.

Odd-parity gauge transformations are generated by a dual vector field $\Xi_{\alpha}=\left(0, \Xi_{A}\right)$ that is expanded as

$$
\Xi_{A}=\sum_{l m} \xi^{l m} X_{A}^{l m}
$$

in which $\xi^{l m}$ depends on the coordinates $x^{a}$ only. Under such a transformation the perturbation quantities change according to (see Appendix $\mathrm{B}$ )

$$
\begin{aligned}
& h_{a} \rightarrow h_{a}^{\prime}=h_{a}-\nabla_{a} \xi+\frac{2}{r} r_{a} \xi, \\
& h_{2} \rightarrow h_{2}^{\prime}=h_{2}-2 \xi
\end{aligned}
$$

where we have discarded the spherical-harmonic labels for brevity (we shall continue with this practice until the end of the section). It is easy to show that the combinations

$$
\tilde{h}_{a}=h_{a}-\frac{1}{2} \nabla_{a} h_{2}+\frac{1}{r} r_{a} h_{2}
$$

are gauge invariant. Equation (5.6) reveals that one can always choose a gauge in which $h_{2}=0$; this is the ReggeWheeler gauge. Equation (5.7) implies that $\tilde{h}_{a}=h_{a}$ in the Regge-Wheeler gauge.

\section{B. Perturbation equations}

The Ricci tensor of the Schwarzschild spacetime vanishes, and as a consequence its perturbation is gauge invariant. Its computation can therefore be carried out in any convenient gauge, and as in the preceding section we shall adopt the Regge-Wheeler gauge. We substitute Eqs. (5.1)- (5.3), having set $h_{2}=0$, into the Ricci tensor of Appendix $\mathrm{B}$. We simplify the result and find that $\delta R_{a b}$ vanishes, $\delta R_{a B}$ is expanded in terms of $X_{B}^{l m}$, and $\delta R_{A B}$ in terms of $X_{A B}^{l m}$. From the Ricci tensor we compute the Einstein tensor and set the result equal to $8 \pi T^{\alpha \beta}$. Each spherical-harmonic component of the field equations can then be extracted by involving the orthonormality relations (3.4) and (3.9) satisfied by the odd-parity harmonics. At the end of this calculation we take advantage of the fact that $\tilde{h}_{a}=h_{a}$ in the Regge-Wheeler gauge. This allows us to make the substitution $h_{a} \rightarrow \tilde{h}_{a}$ and therefore to express the gauge-invariant Einstein tensor in terms of gauge-invariant quantities.

Our final results are

$$
\begin{aligned}
P_{a}= & -\square \tilde{h}_{a}+\nabla_{a} \nabla_{b} \tilde{h}^{b}+\frac{2}{r}\left(r_{b} \nabla_{a} \tilde{h}^{b}-r_{a} \nabla_{b} \tilde{h}^{b}\right) \\
& -\frac{2}{r^{2}} r_{a} r_{b} \tilde{h}^{b}+\frac{l(l+1)}{r^{2}} \tilde{h}_{a}, \\
P= & \nabla_{a} \tilde{h}^{a},
\end{aligned}
$$

where $\square:=g^{a b} \nabla_{a} \nabla_{b}$. The source terms are

$$
\begin{aligned}
P^{a} & =\frac{16 \pi r^{2}}{l(l+1)} \int T^{a B} \bar{X}_{B}^{l m} d \Omega, \\
P & =\frac{16 \pi r^{4}}{(l-1) l(l+1)(l+2)} \int T^{A B} \bar{X}_{A B}^{l m} d \Omega .
\end{aligned}
$$

In Eqs. (5.10) and (5.11) the stress-energy tensor is imagined to be given in fully contravariant form; $T^{a B}$ and $T^{A B}$ are its relevant components in the spacetime coordinates $\left(x^{a}, \theta^{A}\right)$. In the event where the stress-energy 
tensor would be given in covariant or mixed form, its indices would have to be raised with $\left(g^{a b}, r^{-2} \Omega^{A B}\right)-$ the inverse Schwarzschild metric - before evaluating the source terms. In Eqs. (5.8) and (5.9) all lower-case Latin indices are lowered and raised with $g_{a b}$ and $g^{a b}$, respectively.

The perturbation equations are not all independent. By virtue of the Bianchi identities, or the conservation equation (2.10), they are related by

$$
\nabla_{a} P^{a}+\frac{2}{r} r_{a} P^{a}-\frac{(l-1)(l+2)}{r^{2}} P=0 .
$$

\section{Master equation} by

The Cunningham-Price-Moncrief function is defined

$$
\Psi_{\text {odd }}^{l m}:=\frac{2 r}{(l-1)(l+2)} \varepsilon^{a b}\left(\nabla_{a} \tilde{h}_{b}^{l m}-\frac{2}{r} r_{a} \tilde{h}_{b}^{l m}\right),
$$

where $\varepsilon_{a b}$ is the Levi-Civita tensor on the submanifold $\mathcal{M}^{2}$. Apart from a different normalization factor, this is a covariant generalization of the definition provided by Cunningham, Price, and Moncrief [12], who worked with the usual Schwarzschild coordinates. Our definition (and normalization) agrees with the odd-parity function considered by Jhingan and Tanaka 13], who also worked with the Schwarzschild coordinates, but in the frequency domain. The normalization adopted in Eq. (5.13) will be seen to be convenient when we discuss gravitational radiation at future null infinity (Sec. VI) and at the horizon (Sec. VII); our definition of the odd-parity master function is well adapted to the description of radiation fields. It is noteworthy that it can also be expressed as

$$
\Psi_{\text {odd }}^{l m}:=\frac{2 r}{(l-1)(l+2)} \varepsilon^{a b}\left(\partial_{a} h_{b}^{l m}-\frac{2}{r} r_{a} h_{b}^{l m}\right),
$$

in terms of the original (gauge-dependent) perturbation quantities, and in terms of the partial differentiation operator; the function is nonetheless gauge-invariant and a scalar.

The perturbation equations (5.8) and (5.9) give rise to a wave equation for the function $\Psi_{\text {odd }}$ (we resume our practice of discarding the spherical-harmonic labels). As in the preceding section we simply state the final result: As a consequence of the field equations, the odd-parity master function satisfies the Regge-Wheeler equation

$$
\left(\square-V_{\text {odd }}\right) \Psi_{\text {odd }}=S_{\text {odd }},
$$

with potential

$$
V_{\text {odd }}=\frac{l(l+1)}{r^{2}}-\frac{6 M}{r^{3}}
$$

and source term

$$
S_{\text {odd }}=-\frac{2 r}{(l-1)(l+2)} \varepsilon^{a b} \nabla_{a} P_{b}
$$

The validity of Eqs. (5.14)-(5.16) can be verified by brute-force evaluation of both sides of Eqs. (5.14). The general source term for the Regge-Wheeler equation has never been presented in covariant form in the literature. We display it here for the first time, and note that in the usual Schwarzschild coordinates (and in the frequency domain), $S_{\text {odd }}$ agrees with the source term presented in Eq. (18) of Jhingan and Tanaka [13].

\section{Regge-Wheeler function}

The Cunningham-Price-Moncrief function is a close cousin to the more familiar Regge-Wheeler function [1], whose covariant and gauge-invariant definition is

$$
\Psi_{\mathrm{RW}}^{l m}:=\frac{1}{r} r^{a} \tilde{h}_{a}^{l m} .
$$

As we shall see in Secs. VI and VII the Regge-Wheeler function is not well suited to describe the gravitational radiation field, and in this paper we adopt the function $\Psi_{\text {odd }}^{l m}$ of Eq. (5.13) as the fundamental odd-parity master function. It is straightforward to use the perturbation equations to show that these functions are related by

$$
\Psi_{\mathrm{RW}}=\frac{1}{2} t^{a} \nabla_{a} \Psi_{\mathrm{odd}}+\frac{r}{(l-1)(l+2)} r_{a} P^{a},
$$

where $t^{a}=-\varepsilon^{a b} r_{b}$ is the Killing vector of Eq. (2.7). Outside of sources, and apart from a factor of one-half, the Regge-Wheeler function is the time derivative of the Cunningham-Price-Moncrief function.

The function of Eq. (5.17) also satisfies the ReggeWheeler equation,

$$
\left(\square-V_{\text {odd }}\right) \Psi_{\mathrm{RW}}=S_{\mathrm{RW}},
$$

with the potential of Eq. (5.15) and a new source term given by

$$
S_{\mathrm{RW}}=\frac{1}{r}\left[r^{a}\left(\nabla_{a} P-P_{a}\right)-\frac{2}{r}\left(1-\frac{3 M}{r}\right) P\right] .
$$

This covariant form for the source term is also a new result.

\section{RADIATION AT FUTURE NULL INFINITY}

To examine the gravitational perturbations near future null infinity we adopt the retarded coordinates $(u, r, \theta, \phi)$ and express the two-dimensional Schwarzschild metric in the form of Eq. (2.3). In these coordinates, future null infinity corresponds to taking the limit $r \rightarrow \infty$ keeping $u$ fixed, and our strategy will be to expand the metric perturbations in powers of $r^{-1}$. In asymptotically Cartesian coordinates the radiative part of the metric would scale as $r^{-1}$; transforming to spherical coordinates produces the scalings

$$
p_{a b}^{\mathrm{rad}}=O\left(r^{-1}\right), \quad p_{a B}^{\mathrm{rad}}=O\left(r^{0}\right), \quad p_{A B}^{\mathrm{rad}}=O(r)
$$


for the radiative part of the metric perturbations. Our goal is to isolate this, and to calculate how much energy and angular momentum is carried away by the radiation. We find it advantageous to work in gauge in which

$$
t^{a} p_{a b}=0=t^{a} p_{a B}
$$

where $t^{a}$ is the Killing vector of Eq. (2.7). In spite of the fact that $t^{a}$ is not a null vector (except on the even horizon, which is well outside our domain of consideration), this gauge happens to be a perfectly respectable "radiation gauge."

In this section we take $l \geq 2$; as is well known, the low multipoles $l=0$ and $l=1$ do not contain radiative degrees of freedom. We assume that the matter distribution responsible for the radiation is confined to a bounded volume, and that our domain of consideration is outside this volume; we shall therefore be solving the vacuum field equations.

We begin with the even-parity sector of Sec. IV] The gauge conditions imply $h_{u u}=h_{u r}=j_{u}=0$, and the scalings of Eq. (6.1) imply that we are looking for the $r^{-1}$ part of $h_{r r}, K$, and $G$, as well as the $r^{0}$ part of $j_{r}$. These can be determined by expanding the components of the metric perturbation in powers of $r^{-1}$ and substituting them into the field equations of Eqs. (4.13)-(4.16). The expansions for $h_{r r}, K$, and $G$ begin at order $r^{-1}$ and each coefficient is a to-be-determined function of $u$; the expansion for $j_{r}$ begins instead at order $r^{0}$. The field equations return $Q_{a b}, Q_{a}, Q^{b}$, and $Q^{\sharp}$ expanded in powers of $r^{-1}$, and setting each coefficient to zero determines the metric perturbation. We obtain

$$
\begin{aligned}
h_{r r} & =-\frac{l(l+1)}{r^{3}} \int^{u} a\left(u^{\prime}\right) d u^{\prime}+O\left(r^{-4}\right), \\
j_{r} & =\frac{a(u)}{r}+O\left(r^{-2}\right), \\
K & =\frac{l(l+1)}{2 r^{3}} \int^{u} a\left(u^{\prime}\right) d u^{\prime}+O\left(r^{-4}\right), \\
G & =-\frac{2}{(l-1)(l+2)} \frac{\dot{a}(u)}{r}+O\left(r^{-2}\right),
\end{aligned}
$$

where $a(u)$ is a function that is not determined by the vacuum field equations, and $\dot{a}:=d a / d u$. We see that the radiative part of the perturbation is contained entirely in the function $G(u, r)$. With the metric perturbation of Eqs. (6.3) - 6.6) we may evaluate the Zerilli-Moncrief function of Eq. (4.23). The result is

$$
\Psi_{\text {even }}=-\frac{2}{(l-1)(l+2)} \dot{a}(u)+O\left(r^{-1}\right),
$$

and we conclude that the radiative part of the even-parity sector is given by

$$
p_{A B}^{\mathrm{rad}, \text { even }}=r \sum_{l m} \Psi_{\text {even }}^{l m}(u, r=\infty) Y_{A B}^{l m} .
$$

It is obtained by integrating the Zerilli equation 4.25) and evaluating the Zerilli-Moncrief function at $r=\infty$.
We turn next to the odd-parity sector of Sec. $\mathbb{\nabla}$ The gauge conditions imply $h_{u}=0$, and the scalings of Eq. (6.1) imply that we are looking for the $r^{0}$ part of $h_{r}$ and the $r^{1}$ part of $h_{2}$. These are determined by following the same procedure as in the even-parity case, and we obtain

$$
\begin{aligned}
& h_{r}=-\frac{(l-1)(l+2)}{2 r} \int^{u} b\left(u^{\prime}\right) d u^{\prime}+O\left(r^{-2}\right), \\
& h_{2}=b(u) r+O\left(r^{0}\right),
\end{aligned}
$$

where $b(u)$ is not determined by the vacuum field equations. We see that the radiative part of the perturbation is contained entirely in the function $h_{2}(u, r)$. With the metric perturbation of Eqs. (6.9), (6.10) we may evaluate the Cunningham-Price-Moncrief function of Eq. (5.13). The result is

$$
\Psi_{\text {odd }}=b(u)+O\left(r^{-1}\right),
$$

and we conclude that the radiative part of the odd-parity sector is given by

$$
p_{A B}^{\mathrm{rad}, \text { odd }}=r \sum_{l m} \Psi_{\text {odd }}^{l m}(u, r=\infty) X_{A B}^{l m} .
$$

It is obtained by integrating the Regge-Wheeler equation (5.14) and evaluating the Cunningham-Price-Moncrief function at $r=\infty$. Notice that by virtue of Eq. (5.18), the radiative field could instead be expressed in terms of the $u$-integral of the Regge-Wheeler function. The need to perform this integration is inconvenient, and it is the simple relationship of Eq. (6.12) that has motivated the adoption of the Cunningham-Price-Moncrief function as the fundamental odd-parity master function.

The full radiative field is obtained from Eqs. (6.8) and (6.12). We have $p_{a b}^{\mathrm{rad}}=0=p_{a B}^{\mathrm{rad}}$ and

$$
p_{A B}^{\mathrm{rad}}=r \sum_{l m}\left(\Psi_{\text {even }}^{l m} Y_{A B}^{l m}+\Psi_{\mathrm{odd}}^{l m} X_{A B}^{l m}\right),
$$

where $\Psi_{\text {even }}^{l m} \equiv \Psi_{\text {even }}^{l m}(u, r=\infty)$ and $\Psi_{\text {odd }}^{l m} \equiv \Psi_{\text {odd }}^{l m}(u, r=$ $\infty)$. As expected, the radiative field is transverse, and tracefree by virtue of Eqs. (3.11). The two fundamental polarizations of the gravitational wave can be defined by $h_{+}:=p_{\theta \theta} / r^{2}$ and $h_{\times}:=p_{\theta \phi} /\left(r^{2} \sin \theta\right)$. Using the components of the tensorial spherical harmonics listed in Appendix A we obtain

$$
\begin{aligned}
h_{+}= & \frac{1}{r} \sum_{l m}\left\{\Psi_{\text {even }}^{l m}\left[\frac{\partial^{2}}{\partial \theta^{2}}+\frac{1}{2} l(l+1)\right] Y^{l m}\right. \\
& \left.-\Psi_{\text {odd }}^{l m} \frac{i m}{\sin \theta}\left[\frac{\partial}{\partial \theta}-\frac{\cos \theta}{\sin \theta}\right] Y^{l m}\right\}
\end{aligned}
$$

and

$$
\begin{aligned}
h_{\times}= & \frac{1}{r} \sum_{l m}\left\{\Psi_{\text {even }}^{l m} \frac{i m}{\sin \theta}\left[\frac{\partial}{\partial \theta}-\frac{\cos \theta}{\sin \theta}\right] Y^{l m}\right. \\
& \left.+\Psi_{\text {odd }}^{l m}\left[\frac{\partial^{2}}{\partial \theta^{2}}+\frac{1}{2} l(l+1)\right] Y^{l m}\right\} .
\end{aligned}
$$


The energy and angular momentum carried away by the gravitational radiation can be calculated using the techniques developed by Thorne 17]. We compare our Eq. (6.13) to his Eq. (4.3), taking into account the relationship between our tensorial harmonics and his purespin harmonics (this is spelled out in Appendix A). Thorne's mass multipole moments are thus seen to be proportional to $\Psi_{\text {even }}^{l m}(u, r=\infty)$, and his current moments are proportional to $\Psi_{\text {odd }}^{l m}(u, r=\infty)$. Substituting these into Thorne's Eq. (4.16) we obtain

$$
\begin{aligned}
\left\langle\frac{d E}{d u}\right\rangle= & \frac{1}{64 \pi} \sum_{l m}(l-1) l(l+1)(l+2) \\
& \times\left\langle\left|\dot{\Psi}_{\text {even }}^{l m}\right|^{2}+\left|\dot{\Psi}_{\text {odd }}^{l m}\right|^{2}\right\rangle
\end{aligned}
$$

for the averaged rate at which the energy escapes to future null infinity. Substituting instead into Thorne's Eq. (4.23) returns

$$
\begin{aligned}
\left\langle\frac{d J}{d u}\right\rangle= & \frac{1}{64 \pi} \sum_{l m}(l-1) l(l+1)(l+2)(i m) \\
& \times\left\langle\bar{\Psi}_{\text {even }}^{l m} \dot{\Psi}_{\text {even }}^{l m}+\bar{\Psi}_{\text {odd }}^{l m} \dot{\Psi}_{\text {odd }}^{l m}\right\rangle
\end{aligned}
$$

for the averaged rate at which the angular momentum escapes to infinity. This is the component of the angularmomentum vector in the arbitrary $z$ direction which defines the orientation of the angles $\theta$ and $\phi$. The overbar indicates complex conjugation, and it is not difficult to show that $\langle d J / d u\rangle$ is real. The averaging carried out in Eqs. (6.16) and (6.17) is over a characteristic time scale associated with the gravitational wave.

\section{RADIATION AT THE EVENT HORIZON}

To examine the gravitational perturbations near the event horizon we adopt the advanced coordinates $(v, r, \theta, \phi)$ and express the two-dimensional Schwarzschild metric in the form of Eq. 2.4. We want to calculate how much energy and angular momentum is transfered to the black hole by the perturbation, and we shall do so by following the methods devised by Poisson [19]. As in the preceding section we impose the gauge conditions

$$
t^{a} p_{a b}=0=t^{a} p_{a B}
$$

on the metric perturbation; $t^{a}$ is still the Killing vector of Eq. (2.7). Recall that this vector is null on the event horizon, and Eq. (7.1), evaluated at $r=2 M$, is equivalent to Poisson's Eq. (6.5). Poisson then shows that the part of the metric perturbation which is associated with the transport of energy and angular momentum across the horizon is $p_{A B}$. [Refer to Poisson's Eq. (6.10), which establishes the equality between $p_{A B}$ and $\gamma_{A B}^{1}$, the perturbation of the horizon's intrinsic metric.]

In this section we take $l \geq 2$, as the low multipoles $l=0$ and $l=1$ do not contain radiative degrees of freedom.
We assume that the matter distribution responsible for the radiation does not come near the event horizon; we shall therefore be solving the vacuum field equations in an empty neighborhood of the event horizon.

We begin with the even-parity sector of Sec. IV The gauge conditions of Eq. (7.1) imply that $h_{v v}=h_{v r}=$ $j_{v}=0$, so that $h_{r r}, j_{r}, K$, and $G$ are the only nonvanishing components of the metric perturbation. As stated above, the radiation crossing the event horizon is described entirely by $K$ and $G$ evaluated at $r=2 M$. The field equations, however, imply that $K$ vanishes on the horizon. This is verified by expanding $h_{r r}, j_{r}, K$, and $G$ in powers of $f=1-2 M / r$ and substituting them into the field equations of Eqs. (4.13)- 4.16). This calculation reveals also that $G(v, r=2 M)=a(v)$, a function that is not determined by the vacuum field equations. On the other hand, the expansions allow us to evaluate the Zerilli-Moncrief function of Eq. 4.23), and the result is $\Psi_{\text {even }}(r=2 M)=2 M a(v)$. We conclude that on the horizon, the radiative part of the even-parity sector is given by

$$
p_{A B}^{\mathrm{rad}, \text { even }}=2 M \sum_{l m} \Psi_{\mathrm{even}}^{l m}(v, r=2 M) Y_{A B}^{l m} .
$$

It is obtained by integrating the Zerilli equation 4.25 and evaluating the Zerilli-Moncrief function at $r=2 M$.

We turn next to the odd-parity sector of Sec. $\mathbb{\nabla}$ The gauge conditions imply $h_{u}=0$, so that $h_{r}$ and $h_{2}$ are the only nonvanishing components of the metric perturbation. The radiation crossing the event horizon is described entirely by $h_{2}$ evaluated at $r=2 M$, and the field equations imply that $b(v):=h_{2}(v, r=2 M)$ remains as an undetermined function. They also imply that $h_{r}(v, r=2 M)=c(v)$, with

$$
c(v):=\frac{(l-1)(l+2)}{8 M^{2}} \int^{v} b\left(v^{\prime}\right) d v^{\prime} .
$$

These statements are verified by expanding $h_{r}$ and $h_{2}$ in powers of $f=1-2 M / r$ and substituting them into Eqs. (5.8) and (5.9). The expansions allow us also to evaluate the Cunningham-Price-Moncrief function of Eq. (5.13), and the result is

$$
\Psi_{\text {odd }}(r=2 M)=-\frac{4 M}{(l-1)(l+2)} \frac{d c}{d v}=-\frac{1}{2 M} b(v) .
$$

We conclude that on the horizon, the radiative part of the odd-parity sector is given by

$$
p_{A B}^{\mathrm{rad}, \text { odd }}=-2 M \sum_{l m} \Psi_{\text {odd }}^{l m}(v, r=2 M) X_{A B}^{l m} .
$$

It is obtained by integrating the Regge-Wheeler equation (5.14) and evaluating the Cunningham-Price-Moncrief function at $r=2 M$. Notice that by virtue of Eq. (5.18), the radiative field could instead be expressed in terms of the $v$-integral of the Regge-Wheeler function; this would 
give rise to Poisson's Eq. (7.3), a less convenient expression.

The full radiative field is obtained by adding Eqs. (7.2) and (7.3). We have

$$
p_{A B}^{\mathrm{rad}}=2 M \sum_{l m}\left(\Psi_{\mathrm{even}}^{l m} Y_{A B}^{l m}-\Psi_{\mathrm{odd}}^{l m} X_{A B}^{l m}\right),
$$

where $\Psi_{\text {even }}^{l m} \equiv \Psi_{\text {even }}^{l m}(v, r=2 M)$ and $\Psi_{\text {odd }}^{l m} \equiv \Psi_{\text {odd }}^{l m}(v, r=$ $2 M)$. This result should be compared with Eq. (6.13). The rates at which the gravitational perturbation transfers energy and angular momentum to the black hole can now be calculated using the method described in Sec. VII of Poisson [19]. Our Eq. (7.4) replaces his Eq. (7.5), and the rest of the calculation is identical. The final results are

$$
\begin{aligned}
\left\langle\frac{d E}{d v}\right\rangle= & \frac{1}{64 \pi} \sum_{l m}(l-1) l(l+1)(l+2) \\
& \times\left\langle\left|\dot{\Psi}_{\text {even }}^{l m}\right|^{2}+\left|\dot{\Psi}_{\text {odd }}^{l m}\right|^{2}\right\rangle
\end{aligned}
$$

and

$$
\begin{aligned}
\left\langle\frac{d J}{d v}\right\rangle= & \frac{1}{64 \pi} \sum_{l m}(l-1) l(l+1)(l+2)(i m) \\
& \times\left\langle\bar{\Psi}_{\text {even }}^{l m} \dot{\Psi}_{\text {even }}^{l m}+\bar{\Psi}_{\text {odd }}^{l m} \dot{\Psi}_{\text {odd }}^{l m}\right\rangle .
\end{aligned}
$$

These equations replace Poisson's Eqs. (7.8) and (7.9). Notice the similarity between Eqs. (7.5) and (6.16), and between Eqs. (7.6) and (6.17). In Eq. (7.6), $J$ represents the component of the hole's angular-momentum vector in the arbitrary $z$ direction which defines the orientation of the angles $\theta$ and $\phi$. The overbar indicates complex conjugation. The averaging carried out in Eqs. (7.5) and (77.6) is over a characteristic time scale associated with the gravitational perturbation.

\section{LOW MULTIPOLES}

To conclude our presentation of the perturbation formalism we now handle the special cases $l=0$ and $l=1$. Our discussion here will essentially reproduce Zerilli's Appendix G [3], but we will frame it in the language developed in this paper. For concreteness, and for simplicity, we will focus mostly on the task of integrating the vacuum field equations for these low multipoles.

\section{A. Monopole perturbation}

When $l=0$ the only relevant spherical harmonic is $Y^{00}$, which is a constant. It follows that $Y_{A}=X_{A}=$ $Y_{A B}=X_{A B}=0$, and the perturbation is of even parity. The only nonvanishing metric perturbations are

$$
p_{a b}=h_{a b} Y^{00}, \quad p_{A B}=r^{2} K \Omega_{A B} Y^{00} ;
$$

the fields $h_{a}$ and $G$ are not defined. The freedom to perform a gauge transformation is contained in $\Xi_{a}=$ $\xi_{a} Y^{00}, \Xi_{A}=0$, and the perturbations transform as

$$
\begin{aligned}
h_{a b} \rightarrow h_{a b}^{\prime} & =h_{a b}-\nabla_{a} \xi_{b}-\nabla_{b} \xi_{a}, \\
K \rightarrow K^{\prime} & =K-\frac{2}{r} r^{a} \xi_{a} .
\end{aligned}
$$

There is no analogue here of the gauge-invariant quantities $\tilde{h}_{a b}$ and $\tilde{K}$ that were introduced in the general case. The relevant field equations for $h_{a b}$ and $K$ are the $Q_{a b}$ and $Q^{\mathrm{b}}$ equations of Eqs. (4.13) and (4.15), respectively, in which we set $l=0, \tilde{h}_{a b}=h_{a b}$, and $\tilde{K}=K$. The $Q_{a}$ and $Q^{\sharp}$ equations of Eqs. (4.14) and (4.16), respectively, are not defined.

We now solve the perturbation equations in the absence of material sources; in this case $Q_{a b}=0=Q^{b}$. We adopt the advanced coordinates $(v, r, \theta, \phi)$ and the metric of Eq. (2.4). For convenience we rescale the value of $Y^{00}$ and set it equal to unity. We choose $\xi_{a}$ so as to enforce the gauge conditions $h_{r r}=K=0$. This does not fully determine the gauge, because these conditions are preserved under an additional transformation generated by $\xi_{v}=-f \alpha(v)$ and $\xi_{r}=\alpha(v)$, where $f=1-2 M / r$ and $\alpha(v)$ is an arbitrary function. This transformation corresponds to a redefinition of the advanced-time coordinate $v$, of the form $v \rightarrow v^{\prime}=v+\xi^{v}=v+\alpha(v)$, and it changes the nonvanishing perturbations according to $h_{v v} \rightarrow h_{v v}^{\prime}=h_{v v}+2 f \dot{\alpha}(v)$ and $h_{v r} \rightarrow h_{v r}^{\prime}=h_{v r}-\dot{\alpha}(v)$, where an overdot indicates differentiation with respect to $v$.

We now involve the field equations. We notice first that the $Q_{r r}=0$ equation implies that $h_{v r}$ is in fact independent of $r$; since this function of $v$ can be altered at will by a gauge transformation, we may set $h_{v r}=0$. The gauge is now fully determined, and the sole remaining perturbation field is $h_{v v}$. By virtue of the $Q_{v r}=0$ equation we have that $r h_{v v}$ must be a function of $v$ only, but the $Q_{v v}=0$ equation constrains this function to be a constant. We therefore have $h_{v v}=($ constant $) / r$, and this completes the integration of the perturbation equations.

The vacuum, monopole perturbation of a Schwarzschild black hole is given by

$$
p_{v v}=\frac{2 \delta M}{r},
$$

where $\delta M$ is a constant; all other components vanish. This addition to $g_{v v}$ simply shifts the Schwarzschild mass parameter from $M$ to $M+\delta M$. The perturbation must respect Birkhoff's theorem, and the perturbed metric is indeed another Schwarzschild solution.

\section{B. Odd-parity dipole perturbation}

The only surviving odd-parity spherical harmonics for $l=1$ are $X_{A}^{1 m}$, which are obtained from $Y^{1 m}$ using 
Eq. (3.2). The tensorial harmonics $X_{A B}^{1 m}$ vanish, and the only surviving components of the metric perturbation are

$$
p_{a B}=\sum_{m} h_{a}^{1 m} X_{B}^{1 m} .
$$

The perturbations $h_{a}^{1 m}$ can be altered by a gauge transformation generated by $\Xi_{a}=0, \Xi_{A}=\sum_{m} \xi^{1 m} X_{A}^{1 m}$; they change according to

$$
h_{a} \rightarrow h_{a}^{\prime}=h_{a}-\nabla_{a} \xi+\frac{2}{r} r_{a} \xi
$$

There is no analogue here of the gauge-invariant fields $\tilde{h}_{a}$ introduced in Sec. $\nabla$ for the general case. The relevant field equation for $h_{a}$ is the $P_{a}$ equation of Eq. (5.8), in which we set $l=1$ and $\tilde{h}_{a}=h_{a}$. The $P$ equation of Eq. (5.9) is not defined.

We now solve the perturbation equations in the absence of material sources; in this case $P_{a}=0$. We once more adopt the advanced coordinates $(v, r, \theta, \phi)$ and the metric of Eq. (2.4). For convenience we replace the set of complex harmonics $Y^{1 m}$ with the set $Y^{\mathrm{m}}$ given by

$$
\begin{aligned}
\left(Y^{0}, Y^{s}, Y^{c}\right) & =(\cos \theta, \sin \theta \sin \phi, \sin \theta \cos \phi) \\
= & \left(\Omega^{3}, \Omega^{2}, \Omega^{1}\right) .
\end{aligned}
$$

This set is real, but not normalized; summation over $m$ will be replaced by a summation over the abstract index $\mathrm{m}=(0, s, c)$. Notice that $\Omega^{i}$ represents the Cartesian components of a unit radial vector; in ordinary vectorial notation, $\boldsymbol{\Omega}=\boldsymbol{r} / r$, where $\boldsymbol{r}=(x, y, z)$ and $r=|\boldsymbol{r}|$. We shall also need $\Omega_{A}^{i}:=\partial \Omega^{i} / \partial \theta^{A}$, the derivatives of $\Omega^{i}$ with respect to the angles $\theta^{A}$.

We choose $\xi$ so as to enforce the gauge condition $h_{r}=0$. This does not fully determine the gauge, because this condition is preserved under an additional transformation generated by $\xi=\alpha(v) r^{2}$, where $\alpha(v)$ is an arbitrary function. This transformation changes $h_{v}$ according to $h_{v} \rightarrow h_{v}^{\prime}=h_{v}-\dot{\alpha}(v) r^{2}$, where an overdot indicates differentiation with respect to $v$.

We now involve the field equations. We note first that the $P_{r}=0$ equation implies that $h_{v}$ must have the form of $h_{v}=k_{1}(v) r^{2}+k_{2}(v) / r$, where $k_{1}$ and $k_{2}$ are arbitrary functions. The first term can be removed by a gauge transformation, and we are left with $h_{v}=k_{2}(v) / r$ and a fully determined gauge. The $P_{v}=0$ equation then implies that $k_{2}$ must in fact be a constant. We therefore have $h_{v}=($ constant $) / r$, and this completes the integration of the perturbation equations.

The vacuum, odd-parity, dipole perturbation of a Schwarzschild black hole is given by

$$
p_{v A}=\frac{2}{r} \sum_{\mathrm{m}}(\delta J)_{\mathrm{m}} X_{A}^{\mathrm{m}},
$$

where $(\delta J)_{\mathrm{m}}$ are the constants identified in the preceding paragraph, and $X_{A}^{\mathrm{m}}=-\varepsilon_{A}^{B} D_{B} Y^{\mathrm{m}}$ are the real oddparity harmonics; all other components of the metric perturbation vanish. Relating the spherical harmonics to the unit radial vector $\Omega^{i}$ as in Eq. (8.7), and writing the summation over $\mathrm{m}$ as an implicit summation over $i$, we obtain our final expression,

$$
p_{v A}=-\frac{2}{r} \varepsilon_{A}^{B}(\delta J)_{i} \Omega_{B}^{i} .
$$

To give an interpretation to this result we perform a transformation from the spherical coordinates $\left(r, \theta^{A}\right)$ to the Cartesian coordinates $x^{i}=r \Omega^{i}$. The metric perturbation transforms as $p_{v i}=r^{-1} p_{v A} \Omega_{i}^{A}$, where $\Omega_{i}^{A}:=$ $\Omega^{A B} \delta_{i j} \Omega_{B}^{j}$. This gives $p_{v i}=-2 r^{-2} \varepsilon_{A B} \Omega_{i}^{A} \Omega_{j}^{B}(\delta J)^{j}$. We now involve the easily-established identity $\varepsilon_{A B} \Omega_{i}^{A} \Omega_{j}^{B}=$ $\varepsilon_{i j k} \Omega^{k}$, where $\varepsilon_{i j k}$ is the permutation symbol (a fully antisymmetric tensor with entries $-1,0$, or 1 ). This gives

$$
p_{v i}=-\frac{2}{r^{2}} \varepsilon_{i j k}(\delta J)^{j} \Omega^{k}=-\frac{2}{r^{3}}(\boldsymbol{\delta} \boldsymbol{J} \times \boldsymbol{r})_{i} .
$$

This form for the metric tensor allows us to identify $\boldsymbol{\delta} \boldsymbol{J}$ with the angular-momentum vector of the spacetime. The perturbed metric therefore describes a slowly rotating black hole; it is the Kerr metric linearized with respect to its angular-momentum parameter.

\section{Even-parity dipole perturbation}

The even-parity spherical harmonics for $l=1$ are $Y^{1 m}$, $Y_{A}^{1 m}$, and $\Omega_{A B} Y^{1 m} ; Y_{A B}^{1 m}$ vanishes. The metric perturbation is then

$$
\begin{aligned}
p_{a b} & =\sum_{m} h_{a b}^{1 m} Y^{1 m} \\
p_{a B} & =\sum_{m} j_{a}^{1 m} Y_{B}^{1 m} \\
p_{A B} & =r^{2} \Omega_{A B} \sum_{m} K^{1 m} Y^{1 m}
\end{aligned}
$$

and the fields $G^{1 m}$ are not defined. Gauge transformations are generated by $\Xi_{a}=\sum_{m} \xi_{a}^{1 m} Y^{1 m}$ and $\Xi_{A}=$ $\sum_{m} \xi^{1 m} Y_{A}^{1 m}$. The perturbations change according to

$$
\begin{aligned}
h_{a b} & \rightarrow h_{a b}^{\prime}=h_{a b}-\nabla_{a} \xi_{b}-\nabla_{b} \xi_{a}, \\
j_{a} & \rightarrow j_{a}^{\prime}=j_{a}-\xi_{a}-\nabla_{a} \xi+\frac{2}{r} r_{a} \xi \\
K & \rightarrow K^{\prime}=K+\frac{2}{r^{2}} \xi-\frac{2}{r} r^{a} \xi_{a} .
\end{aligned}
$$

There is no analogue here of the gauge-invariant fields $\tilde{h}_{a b}$ and $\tilde{K}$ introduced in Sec. IV] for the general case. The relevant field equations for $h_{a b}, j_{a}$, and $K$ are the $Q_{a b}$, $Q_{a}, Q^{b}$ equations of Eqs. 4.13)-4.15), in which we set $l=1, \tilde{h}_{a b}=h_{a b}-\nabla_{a} j_{b}-\nabla_{b} j_{a}$, and $\tilde{K}=K-2 r^{-1} r^{a} j_{a}$. The $Q^{\sharp}$ equation of Eq. (4.16) is not defined for $l=1$.

We now solve the vacuum field equations, $Q_{a b}=Q_{a}=$ $Q^{b}=0$. We adopt the advanced coordinates $(v, r, \theta, \phi)$, the real spherical harmonics of Eq. (8.7), and a gauge in 
which $h_{r r}=h_{r}=K=0$. This gauge is preserved under a transformation generated by

$$
\begin{aligned}
\xi_{v} & =\frac{2 M}{r} \alpha_{1}(v)+\alpha_{2}(v) r \\
\xi_{r} & =\alpha_{1}(v), \\
\xi & =\alpha_{1}(v) r+\alpha_{2}(v) r^{2},
\end{aligned}
$$

where $\alpha_{1}(v)$ and $\alpha_{2}(v)$ are arbitrary functions. This has the following effect on the remaining components of the metric perturbation:

$$
\begin{aligned}
h_{v v} \rightarrow h_{v v}^{\prime} & =h_{v v}-2 \dot{\alpha}_{2} r-\frac{2 M}{r}\left(2 \dot{\alpha}_{1}-\alpha_{2}\right)+\frac{2 M}{r^{2}} \alpha_{1}, \\
h_{v r} & \rightarrow h_{v r}^{\prime}=h_{v r}-\left(\dot{\alpha}_{1}+\alpha_{2}\right), \\
j_{v} & \rightarrow j_{v}^{\prime}=j_{v}-\dot{\alpha}_{2} r^{2}-\left(\dot{\alpha}_{1}+\alpha_{2}\right) r-\frac{2 M}{r} \alpha_{1} .
\end{aligned}
$$

The $Q_{r r}=0$ equation implies that $h_{v r}$ is a function of $v$ only, and this component can be set equal to zero by a gauge transformation. The remaining gauge freedom is now restricted by $\alpha_{2}=-\dot{\alpha}_{1}$. The $Q_{r}=0$ equation implies that $j_{v}$ must be of the form $j_{v}=k_{1}(v) r^{2}+k_{2}(v) / r$, where $k_{1}$ and $k_{2}$ are arbitrary functions of $v$. With this information and the $Q_{v}=0$ equation we find that $h_{v v}$ must of the form $h_{v v}=-k_{2} / r^{2}+3 \dot{k}_{2} / r+k_{3}+2 k_{1} r$, where $k_{3}$ is an additional arbitrary function. The $Q_{v r}=0$ equation now reveals that $k_{3}=0$, the $Q_{v v}=0$ equation yields $2 M k_{1}=-\ddot{k}_{2}$, and the task of integrating the perturbation equations is completed. Defining $k(v):=k_{2}(v) /(2 M)$, we have obtained

$$
h_{v v}=-2 \ddot{k}(v) r+\frac{6 M}{r} \dot{k}(v)-\frac{2 M}{r^{2}} k(v)
$$

and

$$
j_{v}=-\ddot{k}(v) r^{2}+\frac{2 M}{r} k(v)
$$

for the nonvanishing perturbation fields. The remaining gauge freedom is contained in

$$
\begin{aligned}
\xi_{v} & =-\dot{\alpha}(v) r+\frac{2 M}{r} \alpha(v), \\
\xi_{r} & =\alpha(v), \\
\xi & =-\dot{\alpha}(v) r^{2}+\alpha(v) r,
\end{aligned}
$$

where we have set $\alpha:=\alpha_{1}$ and $\alpha_{2}=-\dot{\alpha}$. Under this transformation the perturbations change according to

$$
h_{v v} \rightarrow h_{v v}^{\prime}=h_{v v}+2 \ddot{\alpha}(v) r-\frac{6 M}{r} \dot{\alpha}(v)+\frac{2 M}{r^{2}} \alpha(v)
$$

and

$$
j_{v} \rightarrow j_{v}^{\prime}=j_{v}+\ddot{\alpha}(v) r^{2}-\frac{2 M}{r} \alpha(v) .
$$

Setting $\alpha(v)=k(v)$ produces $h_{v v}^{\prime}=j_{v}^{\prime}=0$, and we conclude that the perturbation is pure gauge.
The vacuum, even-parity, dipole perturbation of a Schwarzschild black hole can be presented in a gauge in which the nonvanishing components are

$$
p_{v v}=-2 r \ddot{\alpha}_{i} \Omega^{i}+\frac{6 M}{r} \dot{\alpha}_{i} \Omega^{i}-\frac{2 M}{r^{2}} \alpha_{i} \Omega^{i}
$$

and

$$
p_{v A}=-r^{2} \ddot{\alpha}_{i} \Omega_{A}^{i}+\frac{2 M}{r} \alpha_{i} \Omega_{A}^{i},
$$

where the vector $\boldsymbol{\alpha}(v)$ is an arbitrary function of the advanced-time coordinate $v$. This perturbation, however, can be removed by a gauge transformation generated by

$$
\begin{aligned}
& \Xi_{v}=-r \dot{\alpha}_{i} \Omega^{i}+\frac{2 M}{r} \alpha_{i} \Omega^{i} \\
& \Xi_{r}=\alpha_{i} \Omega^{i} \\
& \Xi_{A}=-r^{2} \dot{\alpha}_{i} \Omega_{A}^{i}+r \alpha_{i} \Omega_{A}^{i} .
\end{aligned}
$$

The perturbed metric is therefore a Schwarzschild solution expressed in a coordinate system which differs from the original system $\left(v, r, \theta^{A}\right)$. These coordinates are not inertial. In the limit $M \rightarrow 0$ the perturbed metric is recognized as the metric of flat spacetime expressed in a light-cone coordinate system centered on an accelerated world line (see Ref. 20]); the acceleration vector is $\ddot{\alpha}_{i}(v)$. For $M \neq 0$ the perturbed metric describes a Schwarzschild black hole moving on "the same" accelerated world line.

\section{Acknowledgments}

This work was supported by the National Science and Engineering Research Council of Canada.

\section{APPENDIX A: COMPONENTS OF THE VECTOR AND TENSOR SPHERICAL HARMONICS, AND RELATIONSHIP WITH OTHER SPHERICAL HARMONICS}

We first list the components of the tensorial harmonics introduced in Sec. III According to Eq. (3.1) we have

$$
\begin{aligned}
Y_{\theta}^{l m} & =\frac{\partial}{\partial \theta} Y^{l m}, \\
Y_{\phi}^{l m} & =\frac{\partial}{\partial \phi} Y^{l m} .
\end{aligned}
$$

According to Eq. 3.2 we have

$$
\begin{aligned}
X_{\theta}^{l m} & =-\frac{1}{\sin \theta} \frac{\partial}{\partial \phi} Y^{l m} \\
X_{\phi}^{l m} & =\sin \theta \frac{\partial}{\partial \theta} Y^{l m} .
\end{aligned}
$$


According to Eq. (3.6) we have

$$
\begin{aligned}
Y_{\theta \theta}^{l m} & =\left[\frac{\partial^{2}}{\partial \theta^{2}}+\frac{1}{2} l(l+1)\right] Y^{l m}, \\
Y_{\theta \phi}^{l m} & =\left[\frac{\partial^{2}}{\partial \theta \partial \phi}-\frac{\cos \theta}{\sin \theta} \frac{\partial}{\partial \phi}\right] Y^{l m}, \\
Y_{\phi \phi}^{l m} & =\left[\frac{\partial^{2}}{\partial \phi^{2}}+\sin \theta \cos \theta \frac{\partial}{\partial \theta}+\frac{1}{2} l(l+1) \sin ^{2} \theta\right] Y^{l m} .
\end{aligned}
$$

And according to Eq. (3.7) we have

$$
\begin{aligned}
& X_{\theta \theta}^{l m}=-\frac{1}{\sin \theta}\left(\frac{\partial^{2}}{\partial \theta \partial \phi}-\frac{\cos \theta}{\sin \theta} \frac{\partial}{\partial \phi}\right) Y^{l m}, \\
& X_{\theta \phi}^{l m}=\frac{1}{2}\left(\sin \theta \frac{\partial^{2}}{\partial \theta^{2}}-\frac{1}{\sin \theta} \frac{\partial^{2}}{\partial \phi^{2}}-\cos \theta \frac{\partial}{\partial \theta}\right) Y^{l m}, \\
& X_{\phi \phi}^{l m}=\left(\sin \theta \frac{\partial^{2}}{\partial \theta \partial \phi}-\cos \theta \frac{\partial}{\partial \phi}\right) Y^{l m} .
\end{aligned}
$$

The tensorial harmonics $Y_{A B}^{l m}$ and $X_{A B}^{l m}$ can be related to the spherical-harmonic functions of spin-weight $s=$ \pm 2 [16]. Let $m_{A}$ and $\bar{m}_{A}$ be a complex orthonormal basis on the unit two-sphere, with $m_{A}=2^{-1 / 2}(1, i \sin \theta)$. The relationship is then

$$
\begin{aligned}
Y_{A B}^{l m}= & \frac{1}{2} \sqrt{(l-1) l(l+1)(l+2)} \\
& \times\left({ }_{-2} Y^{l m} m_{A} m_{B}+{ }_{2} Y^{l m} \bar{m}_{A} \bar{m}_{B}\right)
\end{aligned}
$$

and

$$
\begin{aligned}
X_{A B}^{l m}= & -\frac{i}{2} \sqrt{(l-1) l(l+1)(l+2)} \\
& \times\left({ }_{-2} Y^{l m} m_{A} m_{B}-{ }_{2} Y^{l m} \bar{m}_{A} \bar{m}_{B}\right),
\end{aligned}
$$

where ${ }_{s} Y^{l m}$ are the spin-weighted spherical harmonics. These equations can be compared with Eqs. (2.38e) and (2.38f) of Ref. [17]. This reveals that our tensorial harmonics are intimately related to the "pure-spin" harmonics used by Thorne. The relationship is

$$
Y_{A B}^{l m}=\sqrt{\frac{1}{2}(l-1) l(l+1)(l+2)} T_{A B}^{E 2, l m}
$$

and

$$
X_{A B}^{l m}=\sqrt{\frac{1}{2}(l-1) l(l+1)(l+2)} T_{A B}^{B 2, l m} .
$$

Notice that the pure-spin harmonics $T_{A B}^{E 2, l m}$ and $T_{A B}^{B 2, l m}$ are normalized on the unit two-sphere. Our convention here differs from Thorne's, who inserts a factor of $r^{-1}$ in $m_{A}$ and $\bar{m}_{A}$ in order to normalize them on a two-sphere of radius $r$.

\section{APPENDIX B: PERTURBATION OF THE RICCI TENSOR FOR A GENERAL SPHERICALLY-SYMMETRIC BACKGROUND SPACETIME}

The perturbed spacetime metric is written as

$$
{ }^{4} g_{a b}=g_{a b}+p_{a b},
$$

$$
\begin{aligned}
{ }^{4} g_{a B} & =p_{a B}, \\
{ }^{4} g_{A B} & =r^{2} \Omega_{A B}+p_{A B},
\end{aligned}
$$

where $\left(g_{a b}, r^{2} \Omega_{A B}\right)$ are the components of the background metric and $\left(p_{a b}, p_{a B}, p_{A B}\right)$ are the components of the perturbation. In this Appendix we allow the background metric to be completely general, so long as it is spherically symmetric; we do not restrict it to be the Schwarzschild metric. We raise lower-case Latin indices with $g^{a b}$, the inverse to $g_{a b}$, and we raise upper-case Latin indices with $\Omega^{A B}$, the inverse to $\Omega_{A B}$. The inverse perturbed metric is thus

$$
\begin{aligned}
{ }^{4} g^{a b} & =g^{a b}-p^{a b}, \\
{ }^{4} g^{a B} & =-\frac{1}{r^{2}} p^{a B}, \\
{ }^{4} g^{A B} & =\frac{1}{r^{2}} \Omega^{A B}-\frac{1}{r^{4}} p^{A B},
\end{aligned}
$$

up to terms quadratic in the perturbations.

Covariant differentiation with respect to the coordinates $x^{a}$ on the submanifold $\mathcal{M}^{2}$ is indicated with $\nabla_{a}$ : $\nabla_{a} g_{b c}=0$. Covariant differentiation with respect to the coordinates $\theta^{A}$ on the unit two-sphere is indicated with $D_{A}: D_{A} \Omega_{B C}=0$. Quantities which depend only on $x^{a}$ are covariantly constant relative to the connection $\Gamma_{B C}^{A}$; for example $D_{A} r \equiv 0$. Quantities which depend only on $\theta^{A}$ are covariantly constant relative to the connection $\Gamma_{b c}^{a}$; for example $\nabla_{a} \Omega_{A B} \equiv 0$.

A straightforward calculation returns the components of the perturbed connection, which we denote ${ }^{4} \Gamma_{\beta \gamma}^{\alpha}+$ $\delta \Gamma_{\beta \gamma}^{\alpha}$. We obtain

$$
\begin{aligned}
\delta \Gamma_{b c}^{a} & =C_{b c}^{a}, \\
\delta \Gamma_{b C}^{a} & =\frac{1}{2}\left(D_{C} p_{b}^{a}+\nabla_{b} p_{C}^{a}-\nabla^{a} p_{b C}\right)-\frac{1}{r} r_{b} p_{C}^{a}, \\
\delta \Gamma_{B C}^{a} & =\frac{1}{2}\left(D_{B} p_{C}^{a}+D_{C} p_{B}^{a}-\nabla^{a} p_{B C}\right)+r r_{m} \Omega_{B C} p^{a m}, \\
\delta \Gamma_{b c}^{A} & =\frac{1}{2 r^{2}}\left(\nabla_{b} p_{c}{ }^{A}+\nabla_{c} p_{b}{ }^{A}-D^{A} p_{b c}\right), \\
\delta \Gamma_{b C}^{A} & =\frac{1}{2 r^{2}}\left(D_{C} p_{b}{ }^{A}-D^{A} p_{b C}+\nabla_{b} p_{C}^{A}\right)-\frac{1}{r^{3}} r_{b} p_{C}^{A}, \\
\delta \Gamma_{B C}^{A} & =\frac{1}{r^{2}} C_{B C}^{A}+\frac{1}{r} r_{m} \Omega_{B C} p^{m A},
\end{aligned}
$$

where $r_{a}:=\nabla_{a} r$,

$$
C_{b c}^{a}:=\frac{1}{2}\left(\nabla_{c} p_{b}^{a}+\nabla_{b} p_{c}^{a}-\nabla^{a} p_{b c}\right),
$$

and

$$
C_{B C}^{A}:=\frac{1}{2}\left(D_{C} p_{B}^{A}+D_{B} p_{C}^{A}-D^{A} p_{B C}\right) .
$$

The Ricci tensor for the perturbed spacetime is equal to the background Ricci tensor $R_{\alpha \beta}$ plus its perturbation $\delta R_{\alpha \beta}$. We obtain 


$$
\begin{aligned}
\delta R_{a b}= & \nabla_{m} C_{a b}^{m}+\frac{2}{r} r_{m} C_{a b}^{m}-\frac{1}{2} \nabla_{a} \nabla_{b} p_{m}^{m}-\frac{1}{2 r^{2}} D^{M} D_{M} p_{a b}+\frac{1}{2 r^{2}} D_{M}\left(\nabla_{a} p_{b}{ }^{M}+\nabla_{b} p_{a}{ }^{M}\right) \\
& -\frac{1}{2 r^{2}} \nabla_{a} \nabla_{b} p_{M}^{M}+\frac{1}{2 r^{3}}\left(r_{a} \nabla_{b} p_{M}^{M}+r_{b} \nabla_{a} p_{M}^{M}\right)-\frac{1}{r^{4}}\left(r_{a} r_{b}-r \nabla_{a} \nabla_{b} r\right) p_{M}^{M}, \\
\delta R_{a B}= & \frac{1}{2} D_{B}\left(\nabla_{m} p_{a}^{m}-\nabla_{a} p_{m}^{m}+\frac{1}{r} r_{a} p_{m}^{m}\right)-\frac{1}{2}\left(\square p_{a B}-\nabla_{m} \nabla_{a} p_{B}^{m}\right)-\frac{1}{r}\left(r_{a} \nabla_{m} p_{B}^{m}-r_{m} \nabla_{a} p_{B}^{m}\right) \\
& -\frac{1}{r^{2}}\left(r_{a} r_{m}+r \nabla_{a} \nabla_{m} r\right) p_{B}^{m}+\frac{1}{2 r^{2}} D^{M}\left(D_{B} p_{a M}-D_{M} p_{a B}\right)+\frac{1}{2 r^{2}} \nabla_{a}\left(D_{M} p_{B}^{M}-D_{B} p_{M}^{M}\right) \\
& -\frac{1}{r^{3}} r_{a}\left(D_{M} p_{B}^{M}-D_{B} p_{M}^{M}\right), \\
\delta R_{A B}= & \Omega_{A B}\left[r r_{m} \nabla_{n}\left(p^{m n}-\frac{1}{2} g^{m n} p_{k}^{k}\right)+\left(r_{m} r_{n}+r \nabla_{m} \nabla_{n} r\right) p^{m n}\right]-\frac{1}{2} D_{A} D_{B} p_{m}^{m}+\frac{1}{2} \nabla_{m}\left(D_{A} p_{B}^{m}+D_{B} p_{A}^{m}\right) \\
& +\frac{1}{r} r_{m} \Omega_{A B} D_{M} p^{m M}-\frac{1}{2} \square p_{A B}+\frac{1}{r^{2}} D_{M} C_{A B}^{M}-\frac{1}{2 r^{2}} D_{A} D_{B} p_{M}^{M}+\frac{1}{r} r^{m} \nabla_{m}\left(p_{A B}-\frac{1}{2} \Omega_{A B} p_{M}^{M}\right) \\
& -\frac{2}{r^{2}} r^{m} r_{m}\left(p_{A B}-\frac{1}{2} \Omega_{A B} p_{M}^{M}\right),
\end{aligned}
$$

where $\square:=g^{a b} \nabla_{a} \nabla_{b}$. These expressions can be simplified by involving Eqs. (2.6) and (2.8) when the background spacetime is the Schwarzschild spacetime.

Under a gauge transformation generated by the dual vector field $\Xi_{\alpha}=\left(\Xi_{a}, \Xi_{A}\right)$, the components of the metric perturbation change according to

$$
\begin{aligned}
p_{a b} & \rightarrow p_{a b}^{\prime}:=p_{a b}-\nabla_{a} \Xi_{b}-\nabla_{b} \Xi_{a}, \\
p_{a B} & \rightarrow p_{a B}^{\prime}:=p_{a B}-\nabla_{a} \Xi_{B}-D_{B} \Xi_{a}+\frac{2}{r} r_{a} \Xi_{B}, \\
p_{A B} & \rightarrow p_{A B}^{\prime}:=p_{A B}-D_{A} \Xi_{B}-D_{B} \Xi_{A}-2 r r^{m} \Xi_{m} \Omega_{A B} .
\end{aligned}
$$

It can be shown that when the background Ricci tensor vanishes, $\delta R_{a b}, \delta R_{a B}$, and $\delta R_{A B}$ are all invariant under this transformation. We use this property in Secs. IV] and $\mathrm{D}$

\section{APPENDIX C: PERTURBATION EQUATIONS IN $(t, r)$ COORDINATES}

In this Appendix the two-dimensional Schwarzschild metric is written in its standard form

$$
d s^{2}=-f d t^{2}+f^{-1} d r^{2},
$$

with $f=1-2 M / r$. In these coordinates the wave operator is given by

$$
\square \Psi=\left(-\frac{1}{f} \frac{\partial^{2}}{\partial t^{2}}+\frac{\partial}{\partial r} f \frac{\partial}{\partial r}\right) \Psi
$$

We set $\lambda:=l(l+1)=\mu+2$ and $\mu:=(l-1)(l+2)=\lambda-2$.

The even-parity perturbations are $h_{t t}, h_{t r}, h_{r r}, j_{t}, j_{r}, K$, and $G$. Under a gauge transformation generated by $\xi_{t}$, $\xi_{r}$, and $\xi$ they change according to $h_{t t} \rightarrow h_{t t}^{\prime}=h_{t t}+\Delta h_{t t}$, etc., with

$$
\begin{aligned}
\Delta h_{t t} & =-2 \frac{\partial}{\partial t} \xi_{t}+\frac{2 M f}{r^{2}} \xi_{r} \\
\Delta h_{t r} & =-\frac{\partial}{\partial r} \xi_{t}-\frac{\partial}{\partial t} \xi_{r}+\frac{2 M}{r^{2} f} \xi_{t} \\
\Delta h_{r r} & =-2 \frac{\partial}{\partial r} \xi_{r}-\frac{2 M}{r^{2} f} \xi_{r} \\
\Delta j_{t} & =-\frac{\partial}{\partial t} \xi-\xi_{t} \\
\Delta j_{r} & =-\frac{\partial}{\partial r} \xi-\xi_{r}+\frac{2}{r} \xi
\end{aligned}
$$




$$
\begin{aligned}
\Delta K & =-\frac{2 f}{r} \xi_{r}+\frac{\lambda}{r^{2}} \xi, \\
\Delta G & =-\frac{2}{r^{2}} \xi .
\end{aligned}
$$

The gauge-invariant fields are

$$
\begin{aligned}
\tilde{h}_{t t} & =h_{t t}-2 \frac{\partial}{\partial t} j_{t}+\frac{2 M f}{r^{2}} j_{r}+r^{2} \frac{\partial^{2}}{\partial t^{2}} G-M f \frac{\partial}{\partial r} G, \\
\tilde{h}_{t r} & =h_{t r}-\frac{\partial}{\partial r} j_{t}-\frac{\partial}{\partial t} j_{r}+\frac{2 M}{r^{2} f} j_{t}+r^{2} \frac{\partial^{2}}{\partial t \partial r} G+\frac{r-3 M}{f} \frac{\partial}{\partial t} G, \\
\tilde{h}_{r r} & =h_{r r}-2 \frac{\partial}{\partial r} j_{r}-\frac{2 M}{r^{2} f} j_{r}+r^{2} \frac{\partial^{2}}{\partial r^{2}} G+\frac{2 r-3 M}{f} \frac{\partial}{\partial r} G, \\
\tilde{K} & =K-\frac{2 f}{r} j_{r}+r f \frac{\partial}{\partial r} G+\frac{\lambda}{2} G .
\end{aligned}
$$

The field equations are

$$
\begin{aligned}
Q^{t t}= & -\frac{\partial^{2}}{\partial r^{2}} \tilde{K}-\frac{3 r-5 M}{r^{2} f} \frac{\partial}{\partial r} \tilde{K}+\frac{f}{r} \frac{\partial}{\partial r} \tilde{h}_{r r}+\frac{(\lambda+2) r+4 M}{2 r^{3}} \tilde{h}_{r r}+\frac{\mu}{2 r^{2} f} \tilde{K}, \\
Q^{t r}= & \frac{\partial^{2}}{\partial t \partial r} \tilde{K}+\frac{r-3 M}{r^{2} f} \frac{\partial}{\partial t} \tilde{K}-\frac{f}{r} \frac{\partial}{\partial t} \tilde{h}_{r r}-\frac{\lambda}{2 r^{2}} \tilde{h}_{t r}, \\
Q^{r r}= & -\frac{\partial^{2}}{\partial t^{2}} \tilde{K}+\frac{(r-M) f}{r^{2}} \frac{\partial}{\partial r} \tilde{K}+\frac{2 f}{r} \frac{\partial}{\partial t} \tilde{h}_{t r}-\frac{f}{r} \frac{\partial}{\partial r} \tilde{h}_{t t}+\frac{\lambda r+4 M}{2 r^{3}} \tilde{h}_{t t}-\frac{f^{2}}{r^{2}} \tilde{h}_{r r}-\frac{\mu f}{2 r^{2}} \tilde{K}, \\
Q^{t}= & \frac{\partial}{\partial t} \tilde{h}_{r r}-\frac{\partial}{\partial r} \tilde{h}_{t r}+\frac{1}{f} \frac{\partial}{\partial t} \tilde{K}-\frac{2 M}{r^{2} f} \tilde{h}_{t r}, \\
Q^{r}= & -\frac{\partial}{\partial t} \tilde{h}_{t r}+\frac{\partial}{\partial r} \tilde{h}_{t t}-f \frac{\partial}{\partial r} \tilde{K}-\frac{r-M}{r^{2} f} \tilde{h}_{t t}+\frac{(r-M) f}{r^{2}} \tilde{h}_{r r}, \\
Q^{b}= & -\frac{\partial^{2}}{\partial t^{2}} \tilde{h}_{r r}+2 \frac{\partial^{2}}{\partial t \partial r} \tilde{h}_{t r}-\frac{\partial^{2}}{\partial r^{2}} \tilde{h}_{t t}-\frac{1}{f} \frac{\partial^{2}}{\partial t^{2}} \tilde{K}+f \frac{\partial^{2}}{\partial r^{2}} \tilde{K}+\frac{2(r-M)}{r^{2} f} \frac{\partial}{\partial t} \tilde{h}_{t r}-\frac{r-3 M}{r^{2} f} \frac{\partial}{\partial r} \tilde{h}_{t t} \\
& -\frac{(r-M) f}{r^{2}} \frac{\partial}{\partial r} \tilde{h}_{r r}+\frac{2(r-M)}{r^{2}} \frac{\partial}{\partial r} \tilde{K}+\frac{\lambda r^{2}-2(2+\lambda) M r+4 M^{2}}{2 r^{4} f^{2}} \tilde{h}_{t t}-\frac{\lambda r^{2}-2 \mu M r-4 M^{2}}{2 r^{4}} \tilde{h}_{r r}, \\
Q^{\sharp}= & \frac{1}{f} \tilde{h}_{t t}-f \tilde{h}_{r r} .
\end{aligned}
$$

The conservation (Bianchi) identities read

$$
\begin{aligned}
& 0=\frac{\partial}{\partial t} Q^{t t}+\frac{\partial}{\partial r} Q^{t r}+\frac{2(r-M)}{r^{2} f} Q^{t r}-\frac{\lambda}{2 r^{2}} Q^{t}, \\
& 0=\frac{\partial}{\partial t} Q^{t r}+\frac{\partial}{\partial r} Q^{r r}+\frac{M f}{r^{2}} Q^{t t}+\frac{2 r-5 M}{r^{2} f} Q^{r r}-\frac{\lambda}{2 r^{2}} Q^{r}-\frac{f}{r} Q^{b}, \\
& 0=\frac{\partial}{\partial t} Q^{t}+\frac{\partial}{\partial r} Q^{r}+\frac{2}{r} Q^{r}+Q^{b}-\frac{\mu}{2 r^{2}} Q^{\sharp} .
\end{aligned}
$$

The Zerilli-Moncrief function is

$$
\Psi_{\text {even }}=\frac{2 r}{\mu+2}\left[\tilde{K}+\frac{2 f}{\Lambda}\left(f \tilde{h}_{r r}-r \frac{\partial}{\partial r} \tilde{K}\right)\right],
$$

where $\Lambda=\mu+6 M / r$, and its source term is

$$
\begin{aligned}
S_{\text {even }}= & \frac{4}{\Lambda} Q^{r}-\frac{1}{r} Q^{\sharp}+\frac{2}{(\mu+2) \Lambda}\left\{2 r^{2} f \frac{\partial}{\partial r}\left(f Q^{t t}-\frac{1}{f} Q^{r r}\right)+\frac{24 M}{\Lambda} Q^{r r}+2 r f Q^{b}\right. \\
& \left.-\frac{r}{\Lambda}\left[\mu(\mu-2)+12(\mu-3) \frac{M}{r}+84 \frac{M^{2}}{r^{2}}\right]\left(f Q^{t t}-\frac{1}{f} Q^{r r}\right)\right\} .
\end{aligned}
$$


The odd-parity perturbations are $h_{t}, h_{r}$, and $h_{2}$. Under a gauge transformation generated by $\xi$ they change according to $h_{t} \rightarrow h_{t}^{\prime}=h_{t}+\Delta h_{t}$, etc., with

$$
\begin{aligned}
\Delta h_{t} & =-\frac{\partial}{\partial t} \xi, \\
\Delta h_{r} & =-\frac{\partial}{\partial r} \xi+\frac{2}{r} \xi, \\
\Delta h_{2} & =-2 \xi .
\end{aligned}
$$

The gauge-invariant fields are

$$
\begin{aligned}
& \tilde{h}_{t}=h_{t}-\frac{1}{2} \frac{\partial}{\partial t} h_{2}, \\
& \tilde{h}_{r}=h_{r}-\frac{1}{2} \frac{\partial}{\partial r} h_{2}+\frac{1}{r} h_{2} .
\end{aligned}
$$

The field equations are

$$
\begin{aligned}
P^{t} & =-\frac{\partial^{2}}{\partial t \partial r} \tilde{h}_{r}+\frac{\partial^{2}}{\partial r^{2}} \tilde{h}_{t}-\frac{2}{r} \frac{\partial}{\partial t} \tilde{h}_{r}-\frac{\lambda r-4 M}{r^{3} f} \tilde{h}_{t}, \\
P^{r} & =\frac{\partial^{2}}{\partial t^{2}} \tilde{h}_{r}-\frac{\partial^{2}}{\partial t \partial r} \tilde{h}_{t}+\frac{2}{r} \frac{\partial}{\partial t} \tilde{h}_{t}+\frac{\mu f}{r^{2}} \tilde{h}_{r} \\
P & =-\frac{1}{f} \frac{\partial}{\partial t} \tilde{h}_{t}+f \frac{\partial}{\partial r} \tilde{h}_{r}+\frac{2 M}{r^{2}} \tilde{h}_{r} .
\end{aligned}
$$

The conservation (Bianchi) identity reads

$$
0=\frac{\partial}{\partial t} P^{t}+\frac{\partial}{\partial r} P^{r}+\frac{2}{r} P^{r}-\frac{\mu}{r^{2}} P .
$$

The Cunningham-Price-Moncrief function is

$$
\Psi_{\text {odd }}=\frac{2 r}{\mu}\left(\frac{\partial}{\partial r} \tilde{h}_{t}-\frac{\partial}{\partial t} \tilde{h}_{r}-\frac{2}{r} \tilde{h}_{t}\right),
$$

and its source term is

$$
S_{\text {odd }}=\frac{2 r}{\mu}\left(\frac{1}{f} \frac{\partial}{\partial t} P^{r}+f \frac{\partial}{\partial r} P^{t}+\frac{2 M}{r^{2}} P^{t}\right) .
$$

\section{APPENDIX D: PERTURBATION EQUATIONS IN $(u, r)$ COORDINATES}

In this Appendix the two-dimensional Schwarzschild metric is written in the form

$$
d s^{2}=-f d u^{2}-2 d u d r,
$$

with $f=1-2 M / r$. In these coordinates the wave operator is given by

$$
\square \Psi=\left(-2 \frac{\partial^{2}}{\partial u \partial r}+\frac{\partial}{\partial r} f \frac{\partial}{\partial r}\right) \Psi .
$$

We set $\lambda:=l(l+1)=\mu+2$ and $\mu:=(l-1)(l+2)=\lambda-2$.

The even-parity perturbations are $h_{u u}, h_{u r}, h_{r r}, j_{u}, j_{r}, K$, and $G$. Under a gauge transformation generated by $\xi_{u}$, $\xi_{r}$, and $\xi$ they change according to $h_{u u} \rightarrow h_{u u}^{\prime}=h_{u u}+\Delta h_{u u}$, etc., with

$$
\begin{aligned}
\Delta h_{u u} & =-2 \frac{\partial}{\partial u} \xi_{u}-\frac{2 M}{r^{2}} \xi_{u}+\frac{2 M f}{r^{2}} \xi_{r}, \\
\Delta h_{u r} & =-\frac{\partial}{\partial r} \xi_{u}-\frac{\partial}{\partial u} \xi_{r}+\frac{2 M}{r^{2}} \xi_{r}
\end{aligned}
$$




$$
\begin{aligned}
\Delta h_{r r} & =-2 \frac{\partial}{\partial r} \xi_{r}, \\
\Delta j_{u} & =-\frac{\partial}{\partial u} \xi-\xi_{u}, \\
\Delta j_{r} & =-\frac{\partial}{\partial r} \xi-\xi_{r}+\frac{2}{r} \xi, \\
\Delta K & =-\frac{2 f}{r} \xi_{r}+\frac{2}{r} \xi_{u}+\frac{\lambda}{r^{2}} \xi, \\
\Delta G & =-\frac{2}{r^{2}} \xi .
\end{aligned}
$$

The gauge-invariant fields are

$$
\begin{aligned}
\tilde{h}_{u u} & =h_{u u}-2 \frac{\partial}{\partial u} j_{u}-\frac{2 M}{r^{2}} j_{u}+\frac{2 M f}{r^{2}} j_{r}+r^{2} \frac{\partial^{2}}{\partial u^{2}} G+M \frac{\partial}{\partial u} G-M f \frac{\partial}{\partial r} G, \\
\tilde{h}_{u r} & =h_{u r}-\frac{\partial}{\partial r} j_{u}-\frac{\partial}{\partial u} j_{r}+\frac{2 M}{r^{2}} j_{r}+r^{2} \frac{\partial^{2}}{\partial u \partial r} G+r \frac{\partial}{\partial u} G-M \frac{\partial}{\partial r} G, \\
\tilde{h}_{r r} & =h_{r r}-2 \frac{\partial}{\partial r} j_{r}+r^{2} \frac{\partial^{2}}{\partial r^{2}} G+2 r \frac{\partial}{\partial r} G, \\
\tilde{K} & =K+\frac{2}{r} j_{u}-\frac{2 f}{r} j_{r}+r f \frac{\partial}{\partial r} G-r \frac{\partial}{\partial u} G+\frac{\lambda}{2} G .
\end{aligned}
$$

The field equations are

$$
\begin{aligned}
Q^{u u}= & -\frac{\partial^{2}}{\partial r^{2}} \tilde{K}-\frac{2}{r} \frac{\partial}{\partial r} \tilde{K}+\frac{1}{r} \frac{\partial}{\partial u} \tilde{h}_{r r}+\frac{f}{r} \frac{\partial}{\partial r} \tilde{h}_{r r}-\frac{2}{r} \frac{\partial}{\partial r} \tilde{h}_{u r}+\frac{\lambda r+4 M}{2 r^{3}} \tilde{h}_{r r}, \\
Q^{u r}= & \frac{\partial^{2}}{\partial u \partial r} \tilde{K}+\frac{2}{r} \frac{\partial}{\partial u} \tilde{K}-\frac{r-M}{r^{2}} \frac{\partial}{\partial r} \tilde{K}-\frac{f}{r} \frac{\partial}{\partial u} \tilde{h}_{r r}+\frac{1}{r} \frac{\partial}{\partial r} \tilde{h}_{u u}+\frac{1}{r^{2}} \tilde{h}_{u u}-\frac{\lambda+4}{2 r^{2}} \tilde{h}_{u r}+\frac{f}{r^{2}} \tilde{h}_{r r}+\frac{\mu}{2 r^{2}} \tilde{K}, \\
Q^{r r}= & -\frac{\partial^{2}}{\partial u^{2}} \tilde{K}-\frac{r-M}{r^{2}} \frac{\partial}{\partial u} \tilde{K}+\frac{(r-M) f}{r^{2}} \frac{\partial}{\partial r} \tilde{K}-\frac{1}{r} \frac{\partial}{\partial u} \tilde{h}_{u u}+\frac{2 f}{r} \frac{\partial}{\partial u} \tilde{h}_{u r}-\frac{f}{r} \frac{\partial}{\partial r} \tilde{h}_{u u} \\
& +\frac{\mu r+4 M}{2 r^{3}} \tilde{h}_{u u}+\frac{2 f}{r^{2}} \tilde{h}_{u r}-\frac{f^{2}}{r^{2}} \tilde{h}_{r r}-\frac{\mu f}{2 r^{2}} \tilde{K}, \\
Q^{u}= & \frac{\partial}{\partial u} \tilde{h}_{r r}-\frac{\partial}{\partial r} \tilde{h}_{u r}+\frac{\partial}{\partial r} \tilde{K}+\frac{2}{r} \tilde{h}_{u r}-\frac{r-M}{r^{2}} \tilde{h}_{r r}, \\
Q^{r}= & -\frac{\partial}{\partial u} \tilde{h}_{u r}+\frac{\partial}{\partial r} \tilde{h}_{u u}+\frac{\partial}{\partial u} \tilde{K}-f \frac{\partial}{\partial r} \tilde{K}-\frac{2(r-M)}{r^{2}} \tilde{h}_{u r}+\frac{(r-M) f}{r^{2}} \tilde{h}_{r r}, \\
Q^{b}= & -\frac{\partial^{2}}{\partial u^{2}} \tilde{h}_{r r}+2 \frac{\partial^{2}}{\partial u \partial r} \tilde{h}_{u r}-\frac{\partial^{2}}{\partial r^{2}} \tilde{h}_{u u}-2 \frac{\partial^{2}}{\partial u \partial r} \tilde{K}+f \frac{\partial^{2}}{\partial r^{2}} \tilde{K}+\frac{r-M}{r^{2}} \frac{\partial}{\partial u} \tilde{h}_{r r}-\frac{2}{r} \frac{\partial}{\partial u} \tilde{K}-\frac{2}{r} \frac{\partial}{\partial r} \tilde{h}_{u u} \\
& +\frac{2(r-M)}{r^{2}} \frac{\partial}{\partial r} \tilde{h}_{u r}-\frac{(r-M) f}{r^{2}} \frac{\partial}{\partial r} \tilde{h}_{r r}+\frac{2(r-M)}{r^{2}} \frac{\partial}{\partial r} \tilde{K}+\frac{\lambda}{r^{2}} \tilde{h}_{u r}-\frac{\lambda r^{2}-2 \mu M r-4 M^{2}}{2 r^{4}} \tilde{h}_{r r}, \\
Q^{\sharp}= & 2 \tilde{h}_{u r}-f \tilde{h}_{r r} .
\end{aligned}
$$

The conservation (Bianchi) identities read

$$
\begin{aligned}
& 0=\frac{\partial}{\partial u} Q^{u u}+\frac{\partial}{\partial r} Q^{u r}-\frac{M}{r^{2}} Q^{u u}+\frac{2}{r} Q^{u r}-\frac{\lambda}{2 r^{2}} Q^{u}+\frac{1}{r} Q^{b}, \\
& 0=\frac{\partial}{\partial u} Q^{u r}+\frac{\partial}{\partial r} Q^{r r}+\frac{M f}{r^{2}} Q^{u u}+\frac{2 M}{r^{2}} Q^{u r}+\frac{2}{r} Q^{r r}-\frac{\lambda}{2 r^{2}} Q^{r}-\frac{f}{r} Q^{b}, \\
& 0=\frac{\partial}{\partial u} Q^{u}+\frac{\partial}{\partial r} Q^{r}+\frac{2}{r} Q^{r}+Q^{b}-\frac{\mu}{2 r^{2}} Q^{\sharp} .
\end{aligned}
$$

The Zerilli-Moncrief function is

$$
\Psi_{\text {even }}=\frac{2 r}{\mu+2}\left[\tilde{K}+\frac{2}{\Lambda}\left(\tilde{h}_{u u}-2 f \tilde{h}_{u r}+f^{2} \tilde{h}_{r r}+r \frac{\partial}{\partial u} \tilde{K}-r f \frac{\partial}{\partial r} \tilde{K}\right)\right],
$$

where $\Lambda=\mu+6 M / r$, and its source term is

$$
S_{\text {even }}=\frac{4}{\Lambda} Q^{r}-\frac{1}{r} Q^{\sharp}+\frac{2}{(\mu+2) \Lambda}\left\{-2 r^{2}\left(\frac{\partial}{\partial u}-f \frac{\partial}{\partial r}\right)\left(f Q^{u u}+2 Q^{u r}\right)+\frac{24 M}{\Lambda} Q^{r r}+2 r f Q^{b}\right.
$$




$$
\left.-\frac{r}{\Lambda}\left[\mu(\mu-2)+12(\mu-3) \frac{M}{r}+84 \frac{M^{2}}{r^{2}}\right]\left(f Q^{u u}+2 Q^{u r}\right)\right\} .
$$

The odd-parity perturbations are $h_{u}, h_{r}$, and $h_{2}$. Under a gauge transformation generated by $\xi$ they change according to $h_{u} \rightarrow h_{u}^{\prime}=h_{u}+\Delta h_{u}$, etc., with

$$
\begin{aligned}
\Delta h_{u} & =-\frac{\partial}{\partial u} \xi, \\
\Delta h_{r} & =-\frac{\partial}{\partial r} \xi+\frac{2}{r} \xi, \\
\Delta h_{2} & =-2 \xi .
\end{aligned}
$$

The gauge-invariant fields are

$$
\begin{aligned}
& \tilde{h}_{u}=h_{u}-\frac{1}{2} \frac{\partial}{\partial u} h_{2}, \\
& \tilde{h}_{r}=h_{r}-\frac{1}{2} \frac{\partial}{\partial r} h_{2}+\frac{1}{r} h_{2} .
\end{aligned}
$$

The field equations are

$$
\begin{aligned}
P^{u} & =-\frac{\partial^{2}}{\partial u \partial r} \tilde{h}_{r}+\frac{\partial^{2}}{\partial r^{2}} \tilde{h}_{u}-\frac{2}{r} \frac{\partial}{\partial u} \tilde{h}_{r}-\frac{2}{r^{2}} \tilde{h}_{u}-\frac{\mu}{r^{2}} \tilde{h}_{r}, \\
P^{r} & =\frac{\partial^{2}}{\partial u^{2}} \tilde{h}_{r}-\frac{\partial^{2}}{\partial u \partial r} \tilde{h}_{u}+\frac{2}{r} \frac{\partial}{\partial u} \tilde{h}_{u}-\frac{\mu}{r^{2}} \tilde{h}_{u}+\frac{\mu f}{r^{2}} \tilde{h}_{r}, \\
P & =-\frac{\partial}{\partial u} \tilde{h}_{r}-\frac{\partial}{\partial r} \tilde{h}_{u}+f \frac{\partial}{\partial r} \tilde{h}_{r}+\frac{2 M}{r^{2}} \tilde{h}_{r} .
\end{aligned}
$$

The conservation (Bianchi) identity reads

$$
0=\frac{\partial}{\partial u} P^{u}+\frac{\partial}{\partial r} P^{r}+\frac{2}{r} P^{r}-\frac{\mu}{r^{2}} P .
$$

The Cunningham-Price-Moncrief function is

$$
\Psi_{\text {odd }}=\frac{2 r}{\mu}\left(\frac{\partial}{\partial r} \tilde{h}_{u}-\frac{\partial}{\partial u} \tilde{h}_{r}-\frac{2}{r} \tilde{h}_{u}\right),
$$

and its source term is

$$
S_{\text {odd }}=\frac{2 r}{\mu}\left(-\frac{\partial}{\partial u} P^{u}+f \frac{\partial}{\partial r} P^{u}+\frac{\partial}{\partial r} P^{r}+\frac{2 M}{r^{2}} P^{u}\right) .
$$

\section{APPENDIX E: PERTURBATION EQUATIONS IN $(v, r)$ COORDINATES}

In this Appendix the two-dimensional Schwarzschild metric is written in the form

$$
d s^{2}=-f d v^{2}+2 d v d r,
$$

with $f=1-2 M / r$. In these coordinates the wave operator is given by

$$
\square \Psi=\left(2 \frac{\partial^{2}}{\partial v \partial r}+\frac{\partial}{\partial r} f \frac{\partial}{\partial r}\right) \Psi .
$$

We set $\lambda:=l(l+1)=\mu+2$ and $\mu:=(l-1)(l+2)=\lambda-2$.

The even-parity perturbations are $h_{v v}, h_{v r}, h_{r r}, j_{v}, j_{r}, K$, and $G$. Under a gauge transformation generated by $\xi_{v}$, $\xi_{r}$, and $\xi$ they change according to $h_{v v} \rightarrow h_{v v}^{\prime}=h_{v v}+\Delta h_{v v}$, etc., with

$$
\Delta h_{v v}=-2 \frac{\partial}{\partial v} \xi_{v}+\frac{2 M}{r^{2}} \xi_{v}+\frac{2 M f}{r^{2}} \xi_{r}
$$




$$
\begin{aligned}
\Delta h_{v r} & =-\frac{\partial}{\partial r} \xi_{v}-\frac{\partial}{\partial v} \xi_{r}-\frac{2 M}{r^{2}} \xi_{r}, \\
\Delta h_{r r} & =-2 \frac{\partial}{\partial r} \xi_{r} \\
\Delta j_{v} & =-\frac{\partial}{\partial v} \xi-\xi_{v} \\
\Delta j_{r} & =-\frac{\partial}{\partial r} \xi-\xi_{r}+\frac{2}{r} \xi \\
\Delta K & =-\frac{2 f}{r} \xi_{r}-\frac{2}{r} \xi_{v}+\frac{\lambda}{r^{2}} \xi \\
\Delta G & =-\frac{2}{r^{2}} \xi .
\end{aligned}
$$

The gauge-invariant fields are

$$
\begin{aligned}
\tilde{h}_{v v} & =h_{v v}-2 \frac{\partial}{\partial v} j_{v}+\frac{2 M}{r^{2}} j_{v}+\frac{2 M f}{r^{2}} j_{r}+r^{2} \frac{\partial^{2}}{\partial v^{2}} G-M \frac{\partial}{\partial v} G-M f \frac{\partial}{\partial r} G, \\
\tilde{h}_{v r} & =h_{v r}-\frac{\partial}{\partial r} j_{v}-\frac{\partial}{\partial v} j_{r}-\frac{2 M}{r^{2}} j_{r}+r^{2} \frac{\partial^{2}}{\partial v \partial r} G+r \frac{\partial}{\partial v} G+M \frac{\partial}{\partial r} G \\
\tilde{h}_{r r} & =h_{r r}-2 \frac{\partial}{\partial r} j_{r}+r^{2} \frac{\partial^{2}}{\partial r^{2}} G+2 r \frac{\partial}{\partial r} G \\
\tilde{K} & =K-\frac{2}{r} j_{v}-\frac{2 f}{r} j_{r}+r f \frac{\partial}{\partial r} G+r \frac{\partial}{\partial v} G+\frac{\lambda}{2} G .
\end{aligned}
$$

The field equations are

$$
\begin{aligned}
Q^{v v}= & -\frac{\partial^{2}}{\partial r^{2}} \tilde{K}-\frac{2}{r} \frac{\partial}{\partial r} \tilde{K}-\frac{1}{r} \frac{\partial}{\partial v} \tilde{h}_{r r}+\frac{f}{r} \frac{\partial}{\partial r} \tilde{h}_{r r}+\frac{2}{r} \frac{\partial}{\partial r} \tilde{h}_{v r}+\frac{\lambda r+4 M}{2 r^{3}} \tilde{h}_{r r}, \\
Q^{v r}= & \frac{\partial^{2}}{\partial v \partial r} \tilde{K}+\frac{2}{r} \frac{\partial}{\partial v} \tilde{K}+\frac{r-M}{r^{2}} \frac{\partial}{\partial r} \tilde{K}-\frac{f}{r} \frac{\partial}{\partial v} \tilde{h}_{r r}-\frac{1}{r} \frac{\partial}{\partial r} \tilde{h}_{v v}-\frac{1}{r^{2}} \tilde{h}_{v v}-\frac{\lambda+4}{2 r^{2}} \tilde{h}_{v r}-\frac{f}{r^{2}} \tilde{h}_{r r}-\frac{\mu}{2 r^{2}} \tilde{K}, \\
Q^{r r}= & -\frac{\partial^{2}}{\partial v^{2}} \tilde{K}+\frac{r-M}{r^{2}} \frac{\partial}{\partial v} \tilde{K}+\frac{(r-M) f}{r^{2}} \frac{\partial}{\partial r} \tilde{K}+\frac{1}{r} \frac{\partial}{\partial v} \tilde{h}_{v v}+\frac{2 f}{r} \frac{\partial}{\partial v} \tilde{h}_{v r}-\frac{f}{r} \frac{\partial}{\partial r} \tilde{h}_{v v} \\
& +\frac{\mu r+4 M}{2 r^{3}} \tilde{h}_{v v}-\frac{2 f}{r^{2}} \tilde{h}_{v r}-\frac{f^{2}}{r^{2}} \tilde{h}_{r r}-\frac{\mu f}{2 r^{2}} \tilde{K}, \\
Q^{v}= & \frac{\partial}{\partial v} \tilde{h}_{r r}-\frac{\partial}{\partial r} \tilde{h}_{v r}-\frac{\partial}{\partial r} \tilde{K}+\frac{2}{r} \tilde{h}_{v r}+\frac{r-M}{r^{2}} \tilde{h}_{r r}, \\
Q^{r}= & -\frac{\partial}{\partial v} \tilde{h}_{v r}+\frac{\partial}{\partial r} \tilde{h}_{v v}-\frac{\partial}{\partial v} \tilde{K}-f \frac{\partial}{\partial r} \tilde{K}+\frac{2(r-M)}{r^{2}} \tilde{h}_{v r}+\frac{(r-M) f}{r^{2}} \tilde{h}_{r r}, \\
Q^{b}= & -\frac{\partial^{2}}{\partial v^{2}} \tilde{h}_{r r}+2 \frac{\partial^{2}}{\partial v \partial r} \tilde{h}_{v r}-\frac{\partial^{2}}{\partial r^{2}} \tilde{h}_{v v}+2 \frac{\partial^{2}}{\partial v \partial r} \tilde{K}+f \frac{\partial^{2}}{\partial r^{2}} \tilde{K}-\frac{r-M}{r^{2}} \frac{\partial}{\partial v} \tilde{h}_{r r}+\frac{2}{r} \frac{\partial}{\partial v} \tilde{K}_{-} \frac{2}{r} \frac{\partial}{\partial r} \tilde{h}_{v v} \\
& -\frac{2(r-M)}{r^{2}} \frac{\partial}{\partial r} \tilde{h}_{v r}-\frac{(r-M) f}{r^{2}} \frac{\partial}{\partial r} \tilde{h}_{r r}+\frac{2(r-M)}{\partial r} \frac{\partial}{\partial r}-\frac{\lambda}{r^{2}} \tilde{h}_{v r}-\frac{\lambda r^{2}-2 \mu M r-4 M^{2}}{2 r^{4}} \tilde{h}_{r r}, \\
Q^{\sharp}= & -2 \tilde{h}_{v r}-f \tilde{h}_{r r} .
\end{aligned}
$$

The conservation (Bianchi) identities read

$$
\begin{aligned}
& 0=\frac{\partial}{\partial v} Q^{v v}+\frac{\partial}{\partial r} Q^{v r}+\frac{M}{r^{2}} Q^{v v}+\frac{2}{r} Q^{v r}-\frac{\lambda}{2 r^{2}} Q^{v}-\frac{1}{r} Q^{b}, \\
& 0=\frac{\partial}{\partial v} Q^{v r}+\frac{\partial}{\partial r} Q^{r r}+\frac{M f}{r^{2}} Q^{v v}-\frac{2 M}{r^{2}} Q^{v r}+\frac{2}{r} Q^{r r}-\frac{\lambda}{2 r^{2}} Q^{r}-\frac{f}{r} Q^{b}, \\
& 0=\frac{\partial}{\partial v} Q^{v}+\frac{\partial}{\partial r} Q^{r}+\frac{2}{r} Q^{r}+Q^{b}-\frac{\mu}{2 r^{2}} Q^{\sharp} .
\end{aligned}
$$

The Zerilli-Moncrief function is

$$
\Psi_{\text {even }}=\frac{2 r}{\mu+2}\left[\tilde{K}+\frac{2}{\Lambda}\left(\tilde{h}_{v v}+2 f \tilde{h}_{v r}+f^{2} \tilde{h}_{r r}-r \frac{\partial}{\partial v} \tilde{K}-r f \frac{\partial}{\partial r} \tilde{K}\right)\right],
$$


where $\Lambda=\mu+6 M / r$, and its source term is

$$
\begin{aligned}
S_{\text {even }}= & \frac{4}{\Lambda} Q^{r}-\frac{1}{r} Q^{\sharp}+\frac{2}{(\mu+2) \Lambda}\left\{2 r^{2}\left(\frac{\partial}{\partial v}+f \frac{\partial}{\partial r}\right)\left(f Q^{v v}-2 Q^{v r}\right)+\frac{24 M}{\Lambda} Q^{r r}+2 r f Q^{b}\right. \\
& \left.-\frac{r}{\Lambda}\left[\mu(\mu-2)+12(\mu-3) \frac{M}{r}+84 \frac{M^{2}}{r^{2}}\right]\left(f Q^{v v}-2 Q^{v r}\right)\right\} .
\end{aligned}
$$

The odd-parity perturbations are $h_{v}, h_{r}$, and $h_{2}$. Under a gauge transformation generated by $\xi$ they change according to $h_{v} \rightarrow h_{v}^{\prime}=h_{v}+\Delta h_{v}$, etc., with

$$
\begin{aligned}
\Delta h_{v} & =-\frac{\partial}{\partial v} \xi, \\
\Delta h_{r} & =-\frac{\partial}{\partial r} \xi+\frac{2}{r} \xi, \\
\Delta h_{2} & =-2 \xi
\end{aligned}
$$

The gauge-invariant fields are

$$
\begin{aligned}
& \tilde{h}_{v}=h_{v}-\frac{1}{2} \frac{\partial}{\partial v} h_{2}, \\
& \tilde{h}_{r}=h_{r}-\frac{1}{2} \frac{\partial}{\partial r} h_{2}+\frac{1}{r} h_{2} .
\end{aligned}
$$

The field equations are

$$
\begin{aligned}
P^{v} & =-\frac{\partial^{2}}{\partial v \partial r} \tilde{h}_{r}+\frac{\partial^{2}}{\partial r^{2}} \tilde{h}_{v}-\frac{2}{r} \frac{\partial}{\partial v} \tilde{h}_{r}-\frac{2}{r^{2}} \tilde{h}_{v}+\frac{\mu}{r^{2}} \tilde{h}_{r}, \\
P^{r} & =\frac{\partial^{2}}{\partial v^{2}} \tilde{h}_{r}-\frac{\partial^{2}}{\partial v \partial r} \tilde{h}_{v}+\frac{2}{r} \frac{\partial}{\partial v} \tilde{h}_{v}+\frac{\mu}{r^{2}} \tilde{h}_{v}+\frac{\mu f}{r^{2}} \tilde{h}_{r} \\
P & =\frac{\partial}{\partial v} \tilde{h}_{r}+\frac{\partial}{\partial r} \tilde{h}_{v}+f \frac{\partial}{\partial r} \tilde{h}_{r}+\frac{2 M}{r^{2}} \tilde{h}_{r} .
\end{aligned}
$$

The conservation (Bianchi) identity reads

$$
0=\frac{\partial}{\partial v} P^{v}+\frac{\partial}{\partial r} P^{r}+\frac{2}{r} P^{r}-\frac{\mu}{r^{2}} P .
$$

The Cunningham-Price-Moncrief function is

$$
\Psi_{\text {odd }}=\frac{2 r}{\mu}\left(\frac{\partial}{\partial r} \tilde{h}_{v}-\frac{\partial}{\partial v} \tilde{h}_{r}-\frac{2}{r} \tilde{h}_{v}\right)
$$

and its source term is

$$
S_{\text {odd }}=\frac{2 r}{\mu}\left(\frac{\partial}{\partial v} P^{v}+f \frac{\partial}{\partial r} P^{v}-\frac{\partial}{\partial r} P^{r}+\frac{2 M}{r^{2}} P^{v}\right) .
$$

[1] T. Regge and J. A. Wheeler, Phys. Rev. 108, 1063 (1957).

[2] C. V. Vishveshwara, Phys. Rev. D 1, 2870 (1970).

[3] F. J. Zerilli, Phys. Rev. D 2, 2141 (1970).

[4] S. Chandrasekhar, The mathematical theory of black holes (Clarendon Press, Oxford, England, 1983).

[5] V. P. Frolov and I. D. Novikov, Black hole physics: Basic concepts and new developments (Kluwer, Dordrecht, The
Netherlands, 1998)

[6] K. Martel, Phys. Rev. D 69, 044025 (2004).

[7] M. Sasaki and H. Tagoshi, Living Rev. Relativity 6 (2004), 6. [Online article]: cited on February 7, 2008, http://www.livingreviews.org/lrr-2003-6.

[8] R. Gleiser, C. Nicasio, R. Price, and J. Pullin, Phys. Rept. 325, 41 (2000).

[9] V. Moncrief, Ann. Phys. (N.Y.) 88, 323 (1974). 
[10] U. H. Gerlach and U. K. Sengupta, Phys. Rev. D 22, 1300 (1980).

[11] O. Sarbach and M. Tiglio, Phys. Rev. D 64, 084016 (2001).

[12] C. T. Cunningham, R. H. Price, and V. Moncrief, Astrophys. J. 224, 643 (1978).

[13] S. Jhingan and T. Tanaka, Phys. Rev. D 67, 104018 (2003).

[14] K. Martel, Ph.d. thesis, University of Guelph (2003).

[15] C. W. Misner, K. S. Thorne, and J. A. Wheeler, Gravi- tation (Freeman, San Francisco, 1973).

[16] J. Goldberg, A. MacFarlane, E. Newman, F. Rohrlich, and E. Sudarshan, J. Math. Phys. 8, 2155 (1967).

[17] K. S. Thorne, Rev. Mod. Phys. 52, 299 (1980).

[18] C. O. Lousto and R. H. Price, Phys. Rev. D 55, 2124 (1997).

[19] E. Poisson, Phys. Rev. D 70, 084044 (2004).

[20] E. Poisson, Phys. Rev. D 69, 084007 (2004). 